Document downloaded from:

http://hdl.handle.net/10251/71180

This paper must be cited as:

Anna Panagopoulou; Vázquez Molina, J.; A. Kyritsis; Monleón Pradas, M.; Vallés Lluch, A.; Gallego Ferrer, G.; P. Pissis (2013). Glass transition and water dynamics in hyaluronic acid hydrogels. Food Biophysics. 8:192-202. doi:10.1007/s11483-013-9295-2.

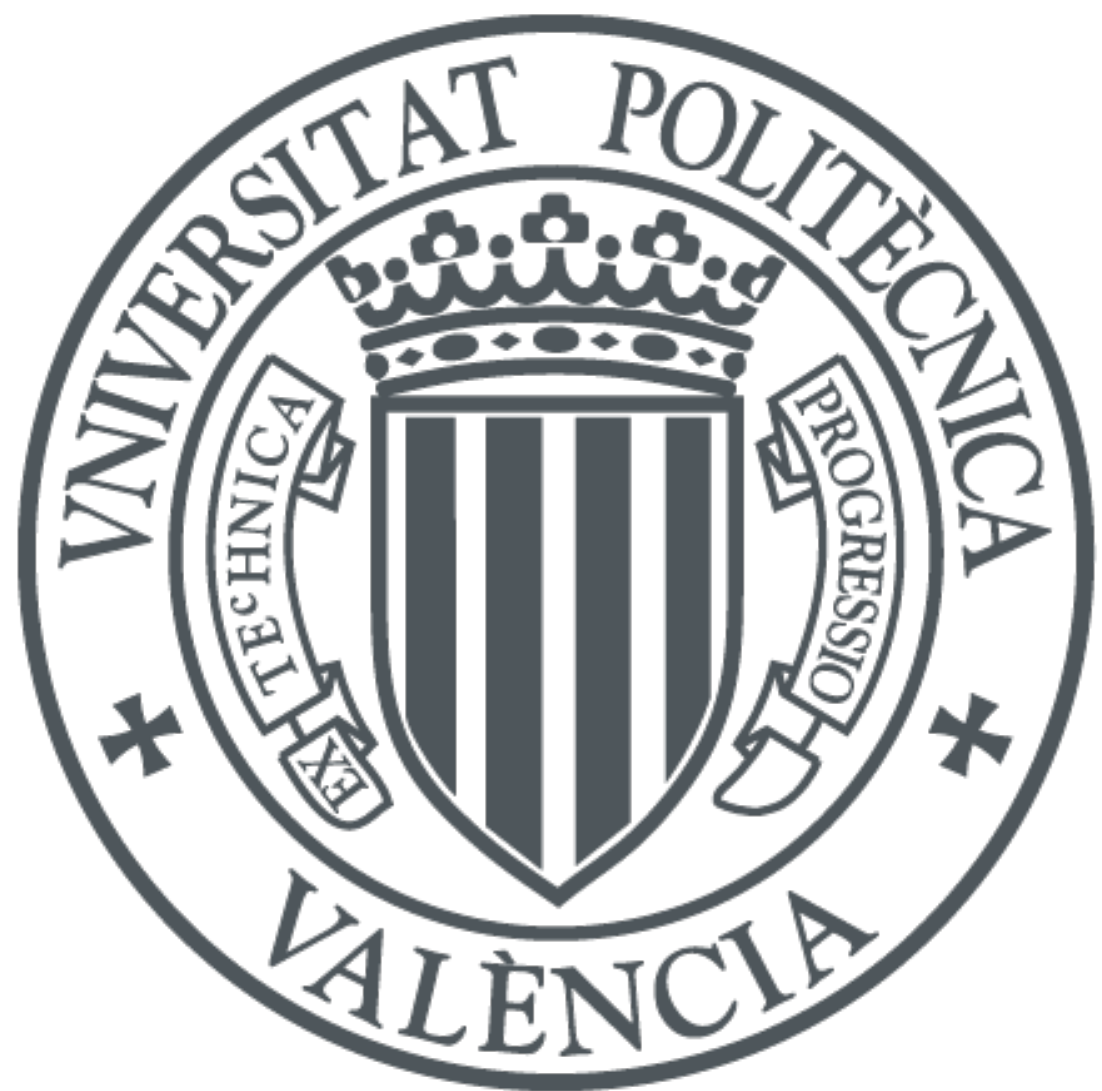

The final publication is available at

http://dx.doi.org/10.1007/s11483-013-9295-2

Copyright Springer Verlag

Additional Information 


\title{
Glass transition and water dynamics in hyaluronic acid hydrogels
}

\author{
Anna Panagopoulou ${ }^{1}$, Joan Vázquez Molina ${ }^{2}$, Apostolos Kyritsis ${ }^{1}$, Manuel \\ Monleón Pradas ${ }^{2,3}$, Anna Vallés Lluch ${ }^{2}$, Gloria Gallego Ferrer ${ }^{2,3}$ and Polycarpos \\ Pissis $^{1}$ \\ ${ }^{1}$ National Technical University of Athens, Zografou Campous, 15780, Athens, Greece \\ ${ }^{2}$ Center for Biomaterials and Tissue Engineering, Universitat Politècnica de València, \\ Camino de Vera s/n, 46022, Valencia, Spain \\ ${ }^{3}$ CIBER en Bioingeniería, Biomateriales y Nanomedicina (CIBER-BBN), Spain
}

\section{Corresponding author}

Anna Panagopoulou

National Technical University of Athens, Department of Physics

Zografou Campus, 15780 Athens, Greece

Tel. +302107722974

Fax. +302107722932

e-mail: panagann@central.ntua.gr, panagann@mail.ntua.gr 


\section{Abstract}

Glass transition and water dynamics in hydrated hyaluronic acid (HA) hydrogels crosslinked by divinyl sulfone (DVS) were studied by differential scanning calorimetry (DSC), dielectric relaxation spectroscopy (DRS) and water sorption desorption (ESI) measurements. A critical water fraction of about $h_{\mathrm{w}}=0.17$ ( $\mathrm{g}$ of water per $g$ of hydrated HA) for a change in the hydration properties of the material was estimated. Water crystallization was recorded by DSC during cooling and heating for water fraction values $h_{\mathrm{w}} \geq 0.31$. The glass transition of the hydrated system was recorded in the water fraction region $0.06 \leq h_{\mathrm{w}} \leq 0.59$. The $T_{\mathrm{g}}$ was found to decrease with increasing hydration level, starting from $T_{\mathrm{g}}=-48^{\circ} \mathrm{C}$ down to about $T_{\mathrm{g}}=-80^{\circ} \mathrm{C}$ and then to stabilize there, for the hydration levels where water crystallization occurs, suggesting that the origin of the glass transition is the combined motion of uncrystallized water molecules attached to primary hydration sites and segments of the HA chains. DRS studies revealed two relaxation peaks, associated with the main secondary relaxation process of uncrystallized water molecules (UCW) triggering the mobility of polar groups and the segmental mobility of HA chains ( $\alpha$ relaxation). The $\alpha$ relaxation was in good agreement with the results by DSC. A qualitative change in the dynamics of the $\alpha$ relaxation was found for $h_{\mathrm{w}}=0.23$ and was attributed to a reorganization of water in the material due to structural changes. Finally, the dielectric strength of the relaxation of UCW was found to decrease in the water fraction region of the structural changes, i.e. for $h_{\mathrm{w}} \sim 0.23$.

Keywords molecular mobility, hydrated hyaluronic acid, hydrogel, uncrystallized water, dielectric relaxation, glass transition 


\section{1) Introduction}

Hyaluronic acid (HA) is a high molecular weight biopolysacharide occurring naturally in all living organisms. It was first isolated as an acid, but under physiological conditions it behaves like a salt (sodium hyaluronate) [1]. In the body it is found in high concentrations in several soft connecting tissues, including skin, umbilical cord, synovial fluid and vitreous humor. Significant amounts of HA are also found in lung, kidney, brain and muscle tissues [2]. In physiological solution, the hyaluronan polymer chain obtains a helical structure, which may be attributed to hydrogen bonding between the hydroxyl groups along the chain. The self association of the HA molecule into a double helical structure has been observed by Atomic Force Microscopy (AFM), even in the case of high molecular weight materials [3]. It is found in the form of an expanded random coil which exhibits high hydrophilicity and may absorb 1000 times its weight in water [4]. It also shows unusual rheological properties depending on the concentration of the solution [2]. HA is one of the most hydrophilic molecules in nature and may be described as nature's moisturizer. For that reason its main biological functions are, among others, the control of tissue hydration and water transport, and to bind water and lubricate movable parts of the body, such as joints and muscles.

HA is used in several biomedical applications due to its high hydrophilicity, biocompatibility and biodegradability. Due to the significance of this molecule for biological procedures a variety of commercially available preparations of HA derivatives [5,6] and cross-linked HA materials have been developed for drug delivery and an extraordinary broad range of biological applications [2]. While linear HA is soluble in water, crosslinked HA forms a hydrogel that can be employed for scaffolds in tissue engineering [7]. Recently, more complex, interpenetrating hydrogel 
networks (IPNs), containing HA, are prepared in order to create scaffolds of advanced properties [8]. For these reasons it becomes clear that the study of the dynamics of water interacting with HA cross-linked materials is of great significance for biomedical applications. The physical but also the hydration properties of HA, in terms of different states of water (free, bound, loosely bound water or crystallized and uncrystallized water (UCW)) in the material, are frequently studied, among other experimental techniques, by differential scanning calorimetry (DSC) [9-11] and (FTIR) [12], while theoretical simulation studies contribute also to the field [13]. Future clinical applications of HA-derived materials critically rely on a more detailed understanding of the effects of HA molecular weight and concentration and will require finely tuned and controllable interactions between HA and its environment [2].

Dielectric measurements contribute to the study of the molecular dynamics of water at biological interfaces, in the case of several biomolecules, such as polysaccharides [14] and hydrated proteins [15-19] and also in the case of synthetic hydrogels [20]. In the case of hydrated proteins, it has been shown that the observed dynamics depends strongly on the hydration level and a minimum amount of water is necessary for the onset of enzymatic activity in globular proteins [21]. The dynamics of water molecules in hydrated proteins which do not form a separate ice phase at subzero temperatures (uncrystallized water), has been extensively studied by dielectric measurements and a main secondary relaxation is recorded, attributed to water molecules in the hydration shell $[16,17,19]$. The main secondary relaxation of uncrystallized water exhibits similar characteristics in various hosting environments $[22,23]$. In the case of studies on hydrated proteins at low levels of hydration, the evolution of this main secondary dielectric relaxation of uncrystallized water with 
increasing hydration level has revealed an interplay between a local dielectric relaxation mode of polar groups on the protein surface interacting with water molecules $[17,19,24,25]$ and a relaxation process originated in the reorientation of water molecules themselves (the main secondary relaxation process of water).

Thermal and dynamic studies of globular hydrated proteins revealed the presence of a thermal glass transition in the temperature range from about -110 to $20^{\circ} \mathrm{C}$, depending on the protein, the hydration level and the experimental technique employed [19,24-32]. The origin of the glass transition of globular hydrated proteins is not fully understood and it is believed to be highly connected to water dynamics. Theoretical studies suggest that the glass transition is driven by the translational reorientation of the hydrogen bonded network on the protein surface [33] and also connect the water-protein glass transition to the denaturation of the globular proteins, suggesting that both correspond to energetic sub-states, while an energy criterion for the onset of mobility of strong protein-water bonds is induced [34]. In the case of globular proteins the observed dielectric relaxation associated to the calorimetric glass transition has been attributed in literature to a combined motion of uncrystallized water molecules in the protein hydration shell and segments of the protein surface $[19,24,25,30]$. Measurements in synthetic hydrogels based on poly(hydroxyl ethyl acrylate) (PHEA) by dielectric and other experimental techniques showed that the segmental $\alpha$ relaxation (dynamic glass transition) is significantly plasticized by water [20]. In addition, correlations were observed between results on the organization of water in the hydrogels and on water effects on polymer dynamics. In particular, distinct changes in the dielectric response at the water content of the completion of the first hydration layer indicated that water molecules themselves contribute to the dielectric response at higher water contents [20]. 
Despite the extended use of HA hydrogels in biological applications, the dielectric behavior of such materials at low water concentrations and subzero temperatures has not been studied so far in detail. In the existing literature dielectric results refer mainly to dilute aqueous solutions of HA salts and particularly at room temperature, using time-domain dielectric measurements [35]. The present study provides experimental dielectric and calorimetric results on HA hydrogels focusing on the low water content regime. In this work, we study the glass transition and the segmental and water molecular dynamics in HA hydrogels, using an experimental approach which combines the DSC technique with dielectric relaxation spectroscopy (DRS) and also with water equilibrium sorption desorption measurements (ESI) at room temperature. The dielectric results are new for HA hydrogels and the combination of the various experimental techniques enable us to follow and evaluate the evolution of the observed dynamics with hydration level. The comparison of the results with similar ones on hydrated proteins may be essential to future application of HA hydrogels in biomedical applications.

\section{2) Materials and Methods}

Sample preparation A $5 \mathrm{wt} \%$ solution of HA (Sigma, HA sodium salt from streptococcus equi sp. $1.63 \mathrm{MDa}$ ) was obtained by stirring the salt in a $0.2 \mathrm{M}$ sodium hydroxide ( $\mathrm{NaOH}$; extrapure, Scharlau) aqueous solution for $24 \mathrm{~h}$. Next, divinyl sulfone (DVS; $118.15 \mathrm{Da}, 97 \%$, Aldrich) was added as crosslinker.at a molar ratio 0.8 and stirred for $1 \mathrm{~min}$ before pouring the mixture into $5 \mathrm{~cm}$ diameter Petri dishes, $5 \mathrm{ml}$ of the mixture each. The solution was allowed to dry for $24 \mathrm{~h}$ at $25^{\circ} \mathrm{C}$. After demolding, the films were rinsed in a 40/60 vol $\%$ mixture of distilled water/acetone 
for 15 min to eliminate $\mathrm{NaOH}$ and DVS residues, followed by three rinsings in distilled water, dried under vacuum and stored.

The hydration of the samples was achieved through equilibration to constant weight (typically achieved in three or four days) above saturated salt solutions in sealed jars [36]. Weights were determined using a Bosch SAE 200 balance with $10^{-4} \mathrm{~g}$ sensitivity. The jars were kept in a dark place, in order to avoid HA degradation due to light exposure.

DSC and DRS measurements, to be described in the following, were performed at various levels of water fraction $h_{w}$, adjusted by equilibration to constant weight above saturated salt solutions, calculated on the wet basis, by

$h_{w}=\frac{m_{\text {water }}}{m}$

where $m$ is the mass of the hydrated sample, the mass of the dry sample and $m_{\text {water }}=m$ $m_{d r y}$ the mass of water inside the sample. The dry mass $m_{d r y}$ was determined by drying the sample in vacuum for $72 \mathrm{~h}$ at $40^{\circ} \mathrm{C}$.

Water equilibrium sorption isotherms (ESI) ESI measurements were performed at $T=25^{\circ} \mathrm{C}$, in controlled nitrogen atmosphere, using a TA Instruments VTI-SA-plus Analyser. The dry mass of the sample was obtained by drying at $T=40^{\circ} \mathrm{C}$. The water activity $\alpha_{w}$ (relative humidity) was systematically varied between about 0.05 and 0.95 in a step of 0.05 . The resulting error in the determination of water content was less than \pm 0.001 .

Differential scanning calorimetry (DSC) For DSC measurements the hydrated samples were placed into aluminum pans at room ambient. After that, the pans with 
Dielectric relaxation spectroscopy (DRS) For DRS measurements [37] the complex dielectric function (known also as dielectric permittivity and dielectric constant), $\varepsilon^{*}(f)=\varepsilon^{\prime}(f)-i \varepsilon^{\prime \prime}(f)$, was determined as a function of frequency, $f,\left(10^{-1}-10^{6} \mathrm{~Hz}\right)$ at constant temperature $\left(-150\right.$ to $20^{\circ} \mathrm{C}$, controlled to better than $\left.\pm 0.1^{\circ} \mathrm{C}\right)$, using a Novocontrol Alpha Analyzer in combination with a Novocontrol Quatro Cryosystem.

Samples for dielectric DRS measurements were in the form of a sheet of an approximate thickness of about $0.2 \mathrm{~mm}$. The hydrated solid samples were placed between two electrodes forming a cylindrical capacitor $12 \mathrm{~mm}$ in diameter.

Dielectric Data Analysis The contribution to the complex permittivity from each relaxation, j, was modelled by an empirical Cole-Cole function:[38]

$$
\varepsilon_{j}^{*}(f, T)=\frac{\Delta \varepsilon_{j}(T)}{1+\left(2 \pi i f \tau_{j}(T)\right)^{\beta_{j}(T)}}
$$

where $\Delta \varepsilon_{j}(T), \tau_{j}(T)$ and $\beta_{j}(T)$ are the dielectric strength, relaxation time and fractional exponent of process $j$, and the contribution of the direct current $(d c)$ to the imaginary part of the complex permittivity by a conductivity term: $\varepsilon^{\prime \prime}(f)=\sigma(T) / \varepsilon_{0}(2 \pi f)$, where $\sigma$ the $d c$ conductivity and $\varepsilon_{0}$ the permittivity of free space. The data analysis software "grafity" [39] was used in the fitting procedure. 


\section{3) Results}

ESI measurements Results of water sorption-desorption measurements, which took place successively at constant temperature, $T=25^{\circ} \mathrm{C}$, are shown in Figure 1: water content $h$ (g of water per $\mathrm{g}$ of dry HA) against water activity $\alpha_{\mathrm{w}}$. Following the sorption data, there is a linear increase of $h$ starting from the dry sample, up to water activity values of about $\alpha_{\mathrm{w}}=0.65$. At that $\alpha_{\mathrm{w}}$ value the water content is around $h=0.2$ (corresponding to water fraction $h_{\mathrm{w}}=0.17$ ). At higher $\alpha_{\mathrm{w}}$ there is a steeper increase of the water content, reaching a maximum value of $h=1.33$ (corresponding to water fraction $h_{\mathrm{w}}=0.57$ ) for $\alpha_{\mathrm{w}}=0.95$. The desorption data exhibit a hysteresis, in the sense that the sample shows higher water content than the respective value during the sorption procedure for the same $\alpha_{\mathrm{w}}$. This hysteresis is evident only in the region of $\alpha_{\mathrm{w}}>0.65$, corresponding to water content $h=0.2$ (on the dry basis, $\mathrm{g}$ of water per $\mathrm{g}$ of dry HA), where a steeper increase in water content was observed during the sorption procedure. The results are in accordance with the work by Servaty etal [12] on the hydration of hyaluronic acid studied by water sorption isotherms and infrared spectroscopy. There, a critical hydration level was estimated, corresponding to a relative humidity value of about $\alpha_{\mathrm{w}}=0.84$. In particular, a steeper increase in water content $h$ was observed in the sorption isotherms for $\alpha_{\mathrm{w}}>0.74$ (similar to the results by ESI in this work) and this was found to be associated with a conformational change in the material, associated to the coverage of the primary hydration sites by water molecules. The onset of this change is believed to be located already to $\alpha_{\mathrm{w}}=0.50$. It was found, that the IR dichroism of the vibrational modes of the pyranose rings vanishes for $\alpha_{\mathrm{w}}>0.84$, and this fact suggests that the helical structure of the HA molecule is diminished due to swelling at high hydration levels, as water molecules 
are condensed near hydrophobic segments. This result is supported by the observed hysteresis for $\alpha_{\mathrm{w}} \geq 0.7$ in Figure 1 . The hysteresis implies that water molecules are not easily detached from the material during desorption possibly because they are condensed near hydrophobic domains.

DSC measurements Cooling and heating scans, both at a rate of $10^{\circ} \mathrm{C} / \mathrm{min}$ were recorded for hydrated HA samples at various hydration levels. Cooling and heating thermograms recorded on various samples, characterized by water fraction values $h_{\mathrm{w}}$ are shown in Figures 2 and 3, respectively. The scale of Figure 2 allows us to observe clearly the crystallization events of water in the samples. No crystallization of water is observed during cooling for $h_{\mathrm{w}} \leq 0.17$. The sample of $h_{\mathrm{w}}=0.31$ exhibits multiple crystallization peaks. Further water increase results in either a single peak (for $h_{\mathrm{w}}=0.42,0.49,0.59$ ) or a double crystallization peak (for $h_{\mathrm{w}}=0.55$ ). In Figure 3 it becomes clear that the samples of $h_{\mathrm{w}} \leq 0.17$ exhibit no cold crystallization of water during heating. At higher $h_{\mathrm{w}}$, the crystallization and melting of water during heating may be observed. In particular, the sample of $h_{\mathrm{w}}=0.31$ shows multiple cold crystallization peaks, starting with a broad peak observed already at temperatures as low as about $T=-70^{\circ} \mathrm{C}$ and a second more pronounced one at higher temperatures, centered at about $T=-20^{\circ} \mathrm{C}$. The exothermic cold crystallization peaks are followed at higher temperature by one endothermic peak due to melting of water, centered at about $T=0^{\circ} \mathrm{C}$. Moving to the sample of $h_{\mathrm{w}}=0.42$, two cold crystallization peaks are observed, centered at about $T=-25$ and $-15^{\circ} \mathrm{C}$ approximately, followed by a melting peak, centered at about $T=0^{\circ} \mathrm{C}$. At higher $h_{\mathrm{w}}$ we observe either one cold crystallization peak (for $h_{\mathrm{w}}=0.49,0.59$ ) or none (for $h_{\mathrm{w}}=0.55$ ). The melting peaks for all the samples are centered at about $T=0^{\circ} \mathrm{C}$. 
In the cooling and heating thermograms in Figures 2 and 3, we may also observe some broad and weak glass transition steps, for $h_{\mathrm{w}} \geq 0.06$, although the scale of the plots makes it hard to observe clearly these steps. For that reason, a magnification in the area of the glass transition during heating for some of the samples is shown in Figure 4. In Figure 4, we observe that the sample of $h_{\mathrm{w}}=0.02$ shows no glass transition step in the experimental temperature range. The glass transition temperature, $T_{\mathrm{g}}$, calculated for the samples of water fraction in the range $0.06<h_{\mathrm{w}}<0.59$ is highlighted in the plot by vertical solid lines. At this point it should be mentioned that the calculation is less trustworthy in the case where cold crystallization interferes in the temperature region of the glass transition. This fact may be observed in Figure 4, regarding the sample of $h_{\mathrm{w}}=0.31$. The glass transition is being interrupted by the broad cold crystallization exothermic peak, the onset of which is located at very low temperature. The same applies also for the samples of water fraction in the range $0.31<h_{\mathrm{w}}<0.59$ (not shown here). Finally, the glass transition is also observed for the sample of the maximum level of hydration studied here, that is for $h_{\mathrm{w}}=0.59$.

The characteristic temperatures calculated by the cooling (crystallization temperature $T_{\mathrm{c}}$ ) and heating thermorgams (melting temperature $T_{\mathrm{m}}$, glass transition temperature $T_{\mathrm{g}}$ ), are plotted in Figure 5 against water fraction $h_{\mathrm{w}}$. The $T_{\mathrm{g}}$ of the hydrated HA initially decreases with water increase, from $T_{\mathrm{g}}=-48^{\circ} \mathrm{C}$ at $h_{\mathrm{w}}=0.06$ to $T_{\mathrm{g}}=-61^{\circ} \mathrm{C}$ at $h_{\mathrm{w}}=0.17$, suggesting that the glass transition of the hydrated system is being plasticized by water molecules. At higher $h_{\mathrm{w}}$ the decreasing $T_{\mathrm{g}}$ value stabilizes. The stabilization of the $T_{\mathrm{g}}$ is not quite clear, due to enhanced scattering of the experimental data in the cold crystallization temperature region, as it has been described earlier. Taking into account the magnitude of the experimental errors, an approximate value of the stabilized $T_{\mathrm{g}}$ has been estimated to $T_{\mathrm{g}}=-80 \pm 5^{\circ} \mathrm{C}$ and the 
position is highlighted in Figure 5 by an horizontal dotted line. Finally, the calculated values of heat capacity step $\Delta C_{\mathrm{p}}$ of the glass transition during heating, are plotted against water fraction $h_{\mathrm{w}}$ in Figure 6, together with the normalized (normalized to the HA mass) $\Delta C_{\mathrm{p}, \mathrm{n}}$ values. The $\Delta C_{\mathrm{p}}$ values initially increase with water fraction increase, from $\Delta C_{\mathrm{p}}=0.53 \mathrm{~J} / \mathrm{g}$ for $h_{\mathrm{w}}=0.06$ to 0.73 for $h_{\mathrm{w}}=0.09$ and then generally decreases with further hydration level increase, down to a value of $\Delta C_{\mathrm{p}}=0.22 \mathrm{~J} / \mathrm{g}$ for $h_{\mathrm{w}}=0.59$. The normalized $\Delta C_{\mathrm{p}, \mathrm{n}}$ values increase initially with hydration increase from $h_{\mathrm{w}}=0.06$ to 0.08 , then decrease again in the range $0.08<h_{\mathrm{w}}<0.4$ (except from the sample of $h_{\mathrm{w}}=0.31$ which, as already mentioned, exhibits enhanced uncertainty regarding the calculations of the glass transition) and then seems to stabilize for $h_{\mathrm{w}}>0.4$. The above mentioned observations will be further evaluated in the discussion section of this paper, together with the rest of the experimental results.

DRS measurements DRS measurements have been performed in HA hydrated hydrogels of water fraction values $h_{\mathrm{w}}=0.06,0.08,0.14$ and 0.23 obtained by equilibration of the samples above saturated salt solution for water activity values $\alpha_{\mathrm{w}}=0.09,0.33,0.54$ and 0.8 , respectively. The frequency dependence of the imaginary part of the dielectric function $\varepsilon^{\prime \prime}$ (dielectric loss) at selected temperatures $T$, indicated on the plot, are shown in Figure 7 for a hydrated HA sample of $h_{\mathrm{w}}=0.08$. Starting at low temperatures, a broad peak is located within the experimental window at $T=-90^{\circ} \mathrm{C}$ centered at about $f=20 \mathrm{~Hz}$ (data (1) in Figure 7). The magnitude of this peak increases with increasing temperature. The time scale of this process and its temperature dependence are similar with those of a relaxation process observed in various hydrated proteins [15-19]. As it will be shown later on, and in accordance to similar interpretations for hydrated proteins, this peak may be associated to a secondary 
relaxation process originated to uncrystallized water molecules (UCW) which are attached onto hydrophilic polar sites on the HA chains. At higher temperatures another peak is observed, centered at about $f=0.6,10$ and $100 \mathrm{~Hz}$ at $T=-45,-30$ and $20^{\circ} \mathrm{C}$, respectively. This peak is probably attributed to the $\alpha$ relaxation of the hydrated system, which is associated to the calorimetric glass transition of the hydrated system. The characteristics of these dielectric relaxations and their evolution with hydration level are the main issues dealt with in this article.

An example of the fitting procedure of the dielectric loss data is shown for a hydrated HA sample of $h_{\mathrm{w}}=0.23$, at temperature $\mathrm{T}=-35^{\circ} \mathrm{C}$ in Figure 8 . The solid line through the experimental dielectric loss data in Figure 8, corresponds to the sum of the calculated contributions to the dielectric loss by a sum of Cole-Cole functions and a conductivity term. At this temperature and for this $h_{\mathrm{w}}$, the maximum of both peaks corresponding to the relaxation of $\mathrm{UCW}$ and the $\alpha$ relaxation are within the experimental window. In Figure 8, the peak of UCW is centered at about $f=0.2 \mathrm{MHz}$ and the $\alpha$ peak at about $600 \mathrm{~Hz}$. At the low frequency side of the experimental window, another contribution is observed, in the same frequency region where the $d c$ conductivity contribution is enhanced. This peak is needed in order to fit the experimental data, and is probably associated to interfacial polarization effects.

The frequency dependence of the dielectric loss $\varepsilon^{\prime \prime}$ at temperature $T=-80^{\circ} \mathrm{C}$, for hydrated HA hydrogels of several hydration levels $h_{\mathrm{w}}$ indicated on the plot, is shown in Figure 9. At this temperature the peak corresponding to the dielectric relaxation of UCW is within the experimental window for all the samples studied. The maximum of the peak is centered at about $10,50,500$ and $10000 \mathrm{~Hz}$ for $h_{\mathrm{w}}=0.06$, $0.08,0.14$ and 0.23 , respectively. This movement of the peak maximum to higher frequencies with the increase of the hydration level, is highlighted in the plot by 
arrows. The magnitude of the peak increases from $h_{\mathrm{w}}=0.06$ to 0.08 , then decreases slightly for 0.14 and finally decreases more profoundly for 0.23 . The frequency dependence of the dielectric loss $\varepsilon^{\prime \prime}$ at temperature $T=-35^{\circ} \mathrm{C}$, for hydrated HA hydrogels of several hydration levels $h_{\mathrm{w}}$ indicated on the plot, is shown in Figure 10. The contributions of the peaks corresponding to the $\alpha$ relaxation of the hydrated system, as calculated by the fittings, are added to the diagram for all of the samples. The $\alpha$ relaxation peak maximum is moving towards higher frequency values as the hydration level increases. The magnitude of the $\alpha$ relaxation increases from $h_{\mathrm{w}}=0.06$ to 0.08 and then decreases again for further increase of the hydration level. Finally, in the low frequency region of the experimental window in Figure 10, we observe an increase in the dielectric loss values with increasing hydration level, which may be attributed to enhanced conductivity contributions and losses due to interfacial polarization effects [30] (see also Figure 7). The fitting parameters $\tau_{j}(T)$ (relaxation time) and $\Delta \varepsilon_{j}(T)$ (dielectric strength) calculated for each process $j$ are plotted against temperature in Figures 11 and 12, respectively, while the parameters $\beta(T)$ for the processes of UCW (uncrystallized water molecules attached to hydrophilic sites of HA) and of the $\alpha$ relaxation are listed in Table 1. The data of Figure 11 for the relaxation of UCW have been expressed by an Arrhenius equation, $f=f_{0} \cdot e^{-\frac{E_{a c t}}{k T}}$, where $E_{a c t}$ and $f_{0}$ are the activation energy and the preexponential factor, respectively. The corresponding values of the activation energy $E_{a c t}$ as well as the logarithm of the pre-exponential factor $\log f_{0}$, are listed in Table 2. The above mentioned results will be evaluated in the upcoming discussion section (section 4). 


\section{4) Discussion}

a relaxation of the hydrated $H A$ hydrogels The thermal glass transition of the hydrated HA hydrogels was recorded by DSC (together with crystallization/melting of water) and the results were described in Figures 5 and 6 (characteristic temperatures, $T_{\mathrm{g}}, T_{\mathrm{c}}, T_{\mathrm{m}}$ and $\left.\Delta C_{\mathrm{p}}\right)$. The $T_{\mathrm{g}}$ of the sample of $h_{\mathrm{w}}=0.06$ is $T_{\mathrm{g}}=-48^{\circ} \mathrm{C}$. This value is relatively low when compared with the one measured by Dynamic Mechanical Analysis (DMA) in the case of hydrated HA hydrogels crosslinked by divinyl sulfone at a molar ratio 50:50 [11], where the glass transition was found at about $T_{\mathrm{g}}=25^{\circ} \mathrm{C}$. This difference could be attributed either to the different crosslinking degree [11] (in our case the molar ratio is 0.8 ), or to differences in level of hydration [11], as well as to the higher frequency of DMA measurements [40]. In Figure 5, it is shown that the recorded glass transition temperature decreases with water fraction starting from the sample of $h_{\mathrm{w}}=0.06$ until a critical water fraction located at about $h_{\mathrm{w}}=0.31$ and seems to stabilize for higher water fraction values at about $T=-80^{\circ} \mathrm{C}$. The stabilization takes place in a water fraction region where water crystallization occurs during cooling (that is for $h_{\mathrm{w}} \geq 0.31$, in the experimental range studied here). This fact implies that the excess water molecules that are able to crystallize forming a separate phase do not further contribute to the plasticization of the glass transition and, consequently, that the glass transition of the hydrated polymeric chains originates to the combined motion of the uncrystallized water molecules attached to hydration sites of the molecular chain and the polymer segments themselves. This stabilization of the $T_{\mathrm{g}}$ has also been observed in hydrated proteins such as lysozyme [24,29], and bovine serum albumin (BSA) [25]. The hydration dependence of the $\alpha$ relaxation associated to the protein glass transition in hydrated BSA has been also studied by dielectric measurements [19], where the evolution was found to be in accordance to calorimetric 
studies on the same system [25]. Interestingly enough, the observed $\alpha$ relaxation of hydrated HA in the present study shows similar characteristics with the observed one in the case of the globular protein BSA. In Figure 11 the temperature dependence of the relaxation times for the $\alpha$ relaxation may be followed. The extrapolation of the trace of the $\alpha$ relaxation to the characteristic relaxation time of DSC (100s [37]) is in accordance to the calorimetric glass transition recorded on similar hydration levels, as it may be seen in Figure 11. The plasticization of the $\alpha$ relaxation is evident as the water fraction increases and the plasticization rate decreases strongly for the sample of water fraction $h_{\mathrm{w}}=0.23$. This $h_{\mathrm{w}}$ value is within the hydration range where the stabilization of the calorimetric glass transition is estimated by DSC (we recall that the determination of the $T_{\mathrm{g}}$ by DSC is uncertain in this region due to interfering cold crystallization events). Regarding the calculated dielectric strength $\Delta \varepsilon$ of the $\alpha$ relaxation, it is shown in Figure 12 that a clear increase of $\Delta \varepsilon$ with temperature decrease (which is typical for the $\alpha$ relaxation of glass forming materials) [23] is observed for all samples studied. $\Delta \varepsilon$ increases when the water fraction increases from $h_{\mathrm{w}}=0.06$ to 0.08 and then decreases again for $h_{\mathrm{w}}=0.14$, in agreement with the evolution of $\Delta C_{\mathrm{p}}$ measured by DSC (Figure 6). At this point, we should make a comparison between the temperature dependence of $\Delta \varepsilon$ for the two samples of $h_{\mathrm{w}}=0.14$ and 0.23 , corresponding to $\alpha_{\mathrm{w}}=0.54$ and 0.80 , respectively. It is obvious in Figure 12, that the temperature dependence of $\Delta \varepsilon$ is differentiated between these two samples, in the sense that the increase is more steep for $h_{\mathrm{w}}=0.14$ and that the trace for $h_{\mathrm{w}}=0.23$ exhibits a curvature which is not observed for the lower water fraction. For this reason, $\Delta \varepsilon$ values are comparable for the two samples at lower temperatures, but are different at higher ones. Another difference between the two samples may be observed in the values of the calculated fractional exponent of the $\alpha$ relaxation $\beta_{\alpha}(T)$ 
in Table 1. The sample of $h_{\mathrm{w}}=0.23$ exhibits a stable value of $\beta_{\alpha}(T)=0.23$ which is relatively low when compared to the values for the rest of the samples which are also temperature dependent for $h_{\mathrm{w}}=0.08$ and 0.14 . These observations imply a qualitative differentiation of the dynamics associated to the $\alpha$ relaxation. These qualitative changes occur in a hydration range where a conformational change in the material is suggested by ESI results of this work, on the basis of the work of Servaty etal [12] (see also section 3, ESI measurements). Probably, there is a critical hydration level above which the domains of helical structure in the material are reduced, because of swelling and the subsequent condensation of water molecules near new accessible hydrophobic segments. The change in the dynamics of the $\alpha$ relaxation of the hydrated HA hydrogels in this particular hydration range supports the idea that the glass transition in biomolecules and globular proteins is qualitatively different than the one observed in the case of less ordered synthetic polymer-water mixtures and might resemble the reorientation of the hydrogen bonded water network interacting with the biopolymer surface. This aspect could also explain the peculiar temperature dependence of the relaxation times of the $\alpha$ relaxation, in the sense that it is not always described by a Vögel-Tamman-Fülcher (VTF) [44] law, e.g samples of $h_{\mathrm{w}}=0.06$ and 0.08 in Figure 11 (an analogous observation was made for BSA in previous studies)[19].

Secondary relaxation due to uncrystallized water molecules attached to primary sorption sites of the HA hydrogels The temperature dependence of the timescale of the secondary relaxation process is shown in Figure 11, for the samples studied in this work. The trace of the relaxation is moving to lower temperatures with water fraction increase, suggesting a plasticizing effect. A similar relaxation process with respect to 
both, the time scale and its temperature dependence has been already observed by employing DRS and Thermally Stimulated Depolarization Currents (TSDC) techniques on various hydrated proteins $[17,19,24,25,45]$ and other biomolecules such as polysaccharides [46,47], seeds [48] and plant tissue [49]. The specific features of this process lead to the interpretation that the observed relaxation process originates presumably to the reorientation of uncrystallized water molecules triggering the motion of small polar groups on the biopolymer surface and not to the reorientation of water molecules themselves. The calculated values of activation energy $E_{\text {act }}$ regarding this relaxation are listed in Table 2. The values are comparable to the reported $E_{\text {act }}$ values for the secondary dielectric relaxation of uncrystallized water, that is near about $0.55 \mathrm{eV}[19,22]$ which corresponds to the energy required to break two hydrogen bonds. More specifically, $E_{\text {act }}$ initially decreases for increasing the water fraction from $h_{\mathrm{w}}=0.06$ to 0.08 and 0.14 and then increases again for $h_{\mathrm{w}}=0.23$ and becomes comparable to the one of the $v$ relaxation of water [23], although its relaxation times are higher (the trace of the $v$ process is shown in Figure 11 as a thick dashed line). The $v$ relaxation is suggested to be the bottom limit for the relaxation times of the main secondary relaxation of uncrystallized water entangled in numerous hydrated biomaterials [23]. The temperature dependence of the dielectric strength $\Delta \varepsilon$ of the relaxation of UCW is shown in Figure 12, where a decrease of $\Delta \varepsilon$ with temperature decrease is evident for all of the samples, resembling the behavior of a secondary dielectric relaxation [23].

Finally, we may follow the evolution of $\Delta \varepsilon$ with increasing water fraction in the hydrogels. In Figure 12, it is shown that the dielectric strength of the secondary relaxation of water initially increases when the water fraction increases from $h_{\mathrm{w}}=0.06$ to 0.08 , then decreases smoothly for 0.14 and finally decreases more steeply for 0.23 . 
We note here that the decrease of the dielectric strength for $h_{\mathrm{w}}=0.23$ is beyond the uncertainty of the experimental data in Figure 12. This is also supported by the observation that the decrease is not universal in the whole temperature range studied, but it is inversed at higher temperature values, where relaxation peaks associated with different relaxation modes are within the experimental window. This finding, i.e. that a relaxation process associated to water molecules exhibits lower dielectric strength in samples with higher water content, is not easily understood. A possible explanation is that at this particular range of hydration levels the distribution of water molecules in the sample changes and different water molecules populations contribute to various relaxation modes. Such a reorganization could be favored by the structural change in the material, which may occurred at this hydration levels as was described in the previous section regarding the $\alpha$ relaxation. Furthermore, we recall that the sample of $h_{\mathrm{w}}=0.23$ shows enhanced contributions to the low frequency dielectric loss, when compared to the rest of the samples, in the high temperature range. This fact may be observed in the raw data in Figure 10 at $T=-35^{\circ} \mathrm{C}$, while it was mentioned during the description of Figure 8 that in the low frequency region of the experimental window an additional peak contributes to the dielectric loss, which might be connected to interfacial polarization effects. These results imply that charge conduction mechanisms change at those hydration levels and support the scenario that the water layer in the hydration shell is being reorganized, as the water molecules diffuse into hydrophobic pockets and the reorientation ability of the HA molecular chains is diminished.

\section{5) Conclusions}


The glass transition and water dynamics in hyaluronic acid (HA) hydrogels crosslinked by divinyl sulfone, at various levels of hydration, have been studied by differential scanning calorimetry (DSC) and dielectric relaxation spectroscopy (DRS). In addition, equilibrium sorption-desorption isotherms (ESI) measurements have been performed at $T=25^{\circ} \mathrm{C}$. The results were discussed in terms of critical levels of hydration and also in relation to similar results on hydrated proteins, other biopolymers and results obtained for HA hydrogels by other experimental techniques.

Several critical hydration levels were determined. For $h_{\mathrm{w}} \leq 0.17$ (g of water per g of hydrated HA) no crystallization of water was observed neither during cooling nor heating. The water molecules at these low levels of hydration are attached onto hydration sites of HA, like in the case of numerous hydrated polymers and biopolymers, and do not form a separate ice phase (uncrystallized water, UCW). The main secondary relaxation process of UCW molecules attached on primary hydration sites of HA was recorded by dielectric measurements, exhibiting an approximate activation energy value of $E_{\text {act }}=0.55 \mathrm{eV}$, corresponding to the energy required to break two hydrogen bonds. The relaxation of UCW was found to become faster with water increase, suggesting that, at low levels of hydration, the relaxation due to UCW contains contributions due to the motion of small polar groups triggered by water molecules.

DSC measurements reveal that water crystallization occurs during cooling, accompanied by cold crystallization during heating for $0.31 \leq h_{\mathrm{w}} \leq 0.59$. The glass transition temperature $T_{\mathrm{g}}$, as recorded by DSC measurements, was found to decrease due to plasticization of the glass transition by water molecules, in the low water fraction region, $h_{\mathrm{w}} \leq 0.17$, where the water molecules are molecularly distributed, UCW. In the hydration range where water crystallization events take place, the 
decrease of glass transition temperature $T_{\mathrm{g}}$ stops, suggesting that the glass transition originates in the combined motion of uncrystallized water molecules at primary sorption sites and segments of the HA chains. The $\alpha$ relaxation associated with the thermal glass transition of the hydrated HA, segmental dynamics, was recorded by DRS, in agreement with DSC results.

Finally, at the critical hydration level of $h_{\mathrm{w}}=0.23$, a qualitative change in the temperature dependence of the dielectric strength of the $\alpha$ relaxation and a reduction of the dielectric strength of the UCW relaxation process were recorded. In addition, during ESI measurements for water activities corresponding to water fractions higher than 0.20 hysteresis effects were observed, between water sorption and desorption processes. These findings support a structural change in the material and a reorganization of water molecules around this critical hydration level. In literature an analogous structural change is attributed to the reduction of the helical structure at high levels of hydration, due to swelling.

HA/water is a very important system showing a very complicated behaviour. In this work we suggest that the combination of ESI, DSC and DRS measurements may help the study of the molecular dynamics in this hydrated biopolymer. The evolution of the observed dynamics in HA hydrogels may be relevant for applications of these materials. Furthermore, the similarities of the observed dynamics to the ones observed in hydrated proteins may facilitate, on the one hand, the synthesis of new materials containing both $\mathrm{HA}$ and protein components and, on the other hand, the better understanding of the role of hydration water in biological function. Regarding the present study, more dielectric experimental data extending to higher levels of hydration, in progress, are expected to provide more information about the organization of water in the system. 


\begin{abstract}
Aknowledgements
This research has been co-financed by the European Union (European Social Fund - ESF) and Greek national funds through the Operational Program "Education and Lifelong Learning" of the National Strategic Reference Framework (NSRF) - Research Funding Program: Heracleitus II. Investing in knowledge society through the European Social Fund (A.P. and P.P.) and Research Funding Program: Aristeia (A.K. and P.P.). A.V.L. and M.M.P. acknowledge financial support of projects MAT2011-28791-C03-02 and -03.

The authors would like to acknowledge Sara Poveda Reyes and María Hernández Palacios for the preparation of the HA sheets.
\end{abstract}

\title{
References
}

[1] T.C. Laurent, In: Ciba foundation symposium 143 1-298, (John Wiley and Sons, New York, (1989))

[2] J. Necas, L. Bartosikov, P. Brauner, J. Kolar, Veterinarni Medicina, 532008 (8): $397-411$.

[3] M. K. Cowman, M. Li, E.A. Balazs, Biophysical Journal 75 (1998) 2030-2037.

[4] M. K. Cowman, S. Matsuoka, Carbohydrate research, 340 (2005) 791-809.

[5] C. E. Schanté, G. Zuber, C. Herlin, T. F. Vendamme, Carbohydrate polymers, 85 (2011) 469-489. 
[6] E. J. Oh, K. Park, K. S. Kim, J. Kim, J-A Yang, J-H. Kong, M. Y. Lee, A. S. Hoffman, S. K. Hahn, Journal of Controlled Release 141 (2010) 2-12.

[7] A.S. Hoffman, Adv. Drug. Deliv. Rev. 54 (2002) 3-12.

[8] F. Lee, M. Kurisawa, Formation and stability of interpenetrating polymer network hydrogels consist of fibrin and hyaluronic acid for tissue engineering, Acta Biomaterialia, (2012).

[9] H. N. Joshi and E. M. Topp, International Journal of Pharmaceutics, 80 (1992) 213-225.

[10] J. Kucerik, A. Prusova, A. Rotaru, K. Flimel, J. Janecek, P. Conte, Thermochimica Acta 523 (2011) 245-249.

[11] M. N. Collins, C. Birkinshaw, J Mater Sci: Mater Med 19 (2008) 3335-3343.

[12] R. Servaty, J. Schiller, H. Binder, K. Arnold, International Journal of Biological Macromolecules 28 (2001) 121-127.

[13] J. Kaufmann, K. Möhle, H. J. Hofmann, K. Arnold, Journal of Molecular Structure (Theochem) 422 (1998) 109-121. 
[14] H. Sugimoto, T. Miki, K. Kanayama, M. Norimoto, J. Non-Cryst. Solids 354 (2008) 3220-3224.

[15] J. Mijović, Y. Bian, R. A. Gross, and B. Chen, Macromolecules 38 (2005) 10812-10819.

[16] J. Swenson, H. Jansson, J. Hedström, and R. Bergman, J. Phys.: Condens. Matter 19 (2007) 205109-205117.

[17] C. Gainaru, A. Fillmer, R. Böhmer, J. Phys. Chem. B 113 (2009) 12628-12631.

[18] W. Doster, S. Busch, A. M. Gaspar, M. S. Appavu, J. Wuttke, H. Scheer, Phys. Rev. Lett. 104 (2010) 098101-098104.

[19] A. Panagopoulou, A. Kyritsis, N. Shinyashiki, P.Pissis, J. Phys. Chem. B 116 (2012) 4593-4602.

[20] P. Pissis, A. Kyritsis, J. Polym. Sci. B: Polym. Phys. 51 (3) (2013) 159-175.

[21] G. Careri, Progress in Biophysics \& Molecular Biology 70 (1998) 223-249.

[22] S. Cerveny, A. Alegria, and J. Colmenero, Physical. Review E 77 (2008) 031803-031807. 
[23] K.L. Ngai, S. Capaccioli, S. Ancherbak, N. Shinyashiki, Phil. Mag. 91 (2011) 1809-1835.

[24] A. Panagopoulou, A. Kyritsis, A. M. Aravantinou, D. Nanopoulos, R. Sabater i Serra, J.L. Gómez Ribellez, N. Shinyashiki, P. Pissis, Food Biophys. 6 (2011) 199209.

[25] A. Panagopoulou, A. Kyritsis, R. Sabater i Serra, J.L. Gómez Ribellez, N. Shinyashiki, P. Pissis, Biochim. Biophys. Acta 1814 (2011) 1984-1996.

[26] R. B. Gregory, Protein-solvent interactions (New York, USA: Marcel Dekker, 1995).

[27] D. Ringe G. A. Petsko, Biophys. Chem. 105 (2003) 667-680.

[28] P. W. Fenimore, H. Frauenfelder, B. H. McMahon, and R. D. Young, Proc. Natl. Acad. Sci. 101 (2004) 14408-14413.

[29] Y. Miyazaki, T. Matsuo, H. Suga, J. Phys. Chem. B 104 (2000) 8044-8052.

[30] N. Shinyashiki, W. Yamamoto, A. Yokoyama, T. Yoshinari, S. Yagihara, K.L. Ngai, S. Capaccioli, J. Phys. Chem. B 113 (2009) 14448- 14456.

[31] S. Khodadadi, A. Malkovskiy, A. Kisliuk, A. P. Sokolov, Biochim. Biophys. Acta 1804 (2010) 15-19. 
[32] H. Jansson, J. Swenson, Biochim. Biophys. Acta 1804 (2010) 20-26.

[33] A. L. Tournier, J. Xu, J. C. Smith, Biophysical J. 85 (2003) 1871-1875.

[34] D. Porter, F. Vollrath, Biochimica et Biophysica Acta 1824 (2012) 785-91.

[35] T. Vuletić, S. Dolanski Babić, T. Ivek, D. Grgičin, and S. Tomić, PHYSICAL REVIEW E 82 (2010) 011922-011932.

[36] L. Greenspan, Humidity Fixed Points of Binary Saturated Aqueous Solutions, J. Res. Nat. Bur. Stand. A Phys. Chem. 81A (1977) 89-96.

[37] F. Kremer, A. Schönhals (Eds.), Broadband Dielectric Spectroscopy (Springer, Berlin, 2002).

[38] R. H. Cole, K. S. Cole, J. Chem. Phys. 10 (1942) 98-105.

[39] http:/grafitylabs.com

[40] R. P. Chartoff, P. T. Weissman, A. Sirkar, The Application of Dynamic Mechanical Methods to $\mathrm{T}_{\mathrm{g}}$ Determination in polymers: An overview, Assignment of the Glass Transition, ASTM STP 1249 Ed. By R.J. Seyler (American Society for Testing and Materials, Philadelphia, 1994) pp. 88-107. 
[41] J. Rault, A. Lucas, R. Neffati, M. Monleon Pradas, Macromolecules 30 (1997) 7866.

[42] M. Salmeron Sanchez, M. Monleon Pradas, J. L. Gomez Ribelles, Journal of Non-Cryst. Solids 307-310 (2002) 750-757.

[43] A.T. Stathopoulos, A. Kyritsis, F.J.R. Colomer, J.L. Gomez Ribelles, N. Shinyashiki, C. Christodoulides, P. Pissis., J. Polym. Sci. B Polym. Phys. 49 (2011) 455-466.

[44] H. Vogel, Physik. Z., 22 (1921) 645-646.

[45] A. Anagnostopoulou-Konsta, P. Pissis, J. Phys. D Appl. Phys. 20 (1987) $1168-1174$.

[46] D. Daoukaki-Diamanti, P. Pissis, G. Boudouris, Chem. Phys. 91 (1984) 315-325.

[47] P. Pissis, J. Phys. D: Appl. Phys. 18 (1985) 1897-1908.

[48] S. Ratkovic, P. Pissis, J. Mater. Sci. 32 (1997) 3061-3068.

[49] P. Pissis, J. Exper. Botany 41 (1990) 677-684. 


\section{Tables}

Table 1. Fractional exponent $\beta(T)$ of the Cole-Cole function for the $\alpha$ relaxation of the hydrated HA hydrogels and the secondary relaxation associated with the reorientational motion of uncrystallized water molecules attached to hydration sites of the HA molecule

\begin{tabular}{|c|c|c|c|c|}
\hline $\boldsymbol{h}_{\mathrm{w}}$ & $\mathbf{0 . 0 6}$ & $\mathbf{0 . 0 8}$ & $\mathbf{0 . 1 4}$ & $\mathbf{0 . 2 3}$ \\
\hline $\boldsymbol{\beta}_{\boldsymbol{\alpha}}(\mathbf{T})$ & 0.52 & $0.52-0.63$ & $0.51-0.99$ & 0.23 \\
\hline $\boldsymbol{\beta}_{\mathrm{UCW}}(\mathbf{T})$ & 0.19 & 0.29 & 0.24 & 0.23 \\
\hline
\end{tabular}

Table 2. Activation Energy, $E_{\text {act }}$, and Logarithm of the Pre-Exponential Factor, $\log f_{0}$, of the Arrhenius Equation for the secondary relaxation associated with the reorientational motion of uncrystallized water molecules attached to hydration sites of the HA molecule

\begin{tabular}{|c|c|c|}
\hline $\boldsymbol{h}_{\mathrm{w}}$ & $\begin{array}{c}\mathbf{E}_{\text {act }} \\
(\mathbf{e V})\end{array}$ & $\log f_{\mathbf{0}}$ \\
\hline $\mathbf{0 . 0 6}$ & $0.61 \pm 0.01$ & $16.34 \pm 0.18$ \\
\hline $\mathbf{0 . 0 8}$ & $0.50 \pm 0.01$ & $14.58 \pm 0.15$ \\
\hline $\mathbf{0 . 1 4}$ & $0.47 \pm 0.01$ & $14.36 \pm 0.28$ \\
\hline $\mathbf{0 . 2 3}$ & $0.58 \pm 0.01$ & $19.09 \pm 0.11$ \\
\hline
\end{tabular}




\section{Figure Captions}

Fig.1 Water content $h_{d}$ against water activity $\alpha_{w}$ at $25^{\circ} \mathrm{C}$ for a HA hydrogel. Solid circles $(\bullet)$ correspond to sorption data and open squares $(\square)$ to data recorded during the desorption procedure, which took place successively after sorption.

Fig.2 Normalized heat flow during cooling (at $10^{\circ} \mathrm{C} / \mathrm{min}$ ) against temperature in hydrated HA hydrogels at different water fractions $h_{w}$ indicated on the plot.

Fig.3 Normalized heat flow during heating (at $10^{\circ} \mathrm{C} / \mathrm{min}$ ) against temperature in hydrated HA hydrogels at different water fractions $h_{w}$ indicated on the plot.

Fig.4 Normalized heat flow during heating (at $10^{\circ} \mathrm{C} / \mathrm{min}$ ) against temperature in hydrated HA hydrogels at selected water fractions $h_{w}$ indicated on the plot. The plot shows a magnification in the region of the glass transition for the selected samples. The glass transition temperature $T_{\mathrm{g}}$, is highlighted by vertical solid lines.

Fig.5 Characteristic temperatures, glass transition Temperature, $T_{\mathrm{g}}(\boldsymbol{\square})$, crystallization temperature, $T_{\mathrm{c}}(\bullet, \nabla, \bullet)$, melting temperature, $T_{\mathrm{m}}(\mathrm{O}, \triangle)$, against water fraction $h_{\mathrm{w}}$ Fig.6 Heat capacity step $\Delta C_{\mathrm{p}}$ of the glass transition of the hydrated hydrogel against water fraction $h_{\mathrm{w}}$. Normalized values to the mass of HA $(\mathrm{O}), \Delta C_{\mathrm{p}, \mathrm{n}}$, are also added to the plot.

Fig.7 Imaginary part of the dielectric function, $\varepsilon^{\prime \prime}$ (dielectric loss), against frequency, at selected temperatures indicated on the plot, for a hydrated HA hydrogel of water fraction $h_{\mathrm{w}}=0.08$ 
Fig.8 Dielectric loss against frequency $f(O)$ for a hydrated HA hydrogel of $h_{\mathrm{w}}=0.23$, 
relaxation of uncrystallized water $(\mathrm{UCW})$ for $h_{\mathrm{w}}=0.06(\square), 0.08(\mathrm{O}), 0.14(\triangle)$ and $0.23(\diamond)$ 
Figures and Captions

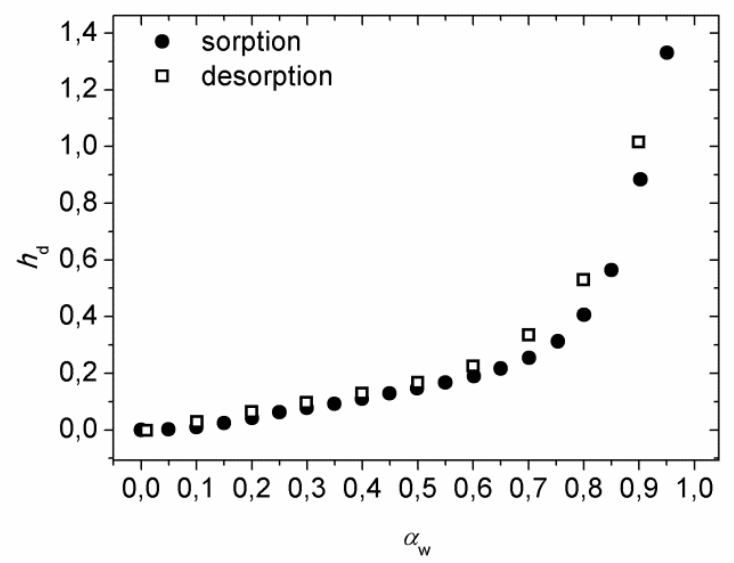

Fig.1 Water content $h_{d}$ against water activity $\alpha_{w}$ at $25^{\circ} \mathrm{C}$ for a HA hydrogel. Solid circles $(\bullet)$ correspond to sorption data and open squares $(\square)$ to data recorded during the desorption procedure, which took place successively after sorption.

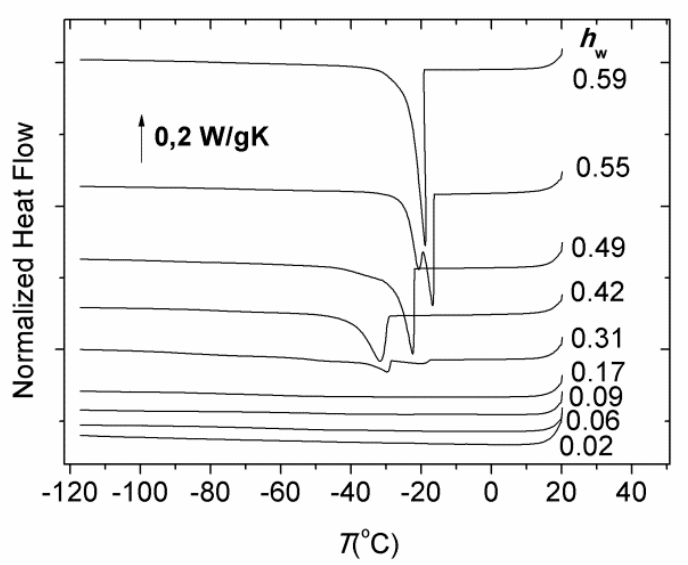

Fig.2 Normalized heat flow during cooling (at $10^{\circ} \mathrm{C} / \mathrm{min}$ ) against temperature in hydrated HA hydrogels at different water fractions $h_{w}$ indicated on the plot. 


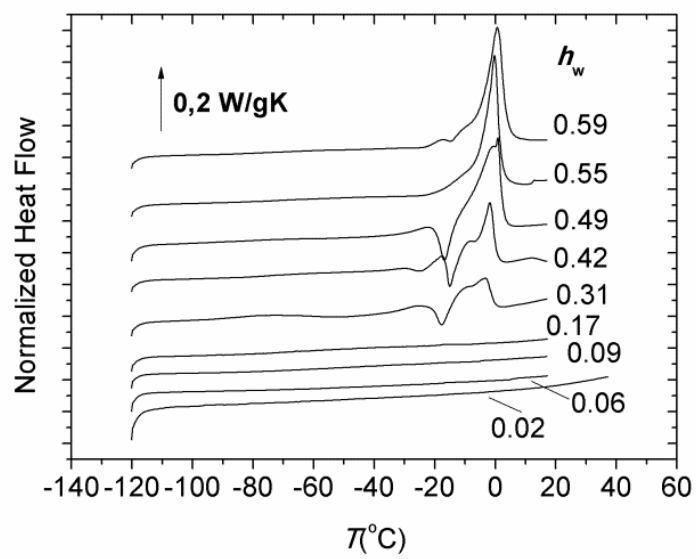

Fig.3 Normalized heat flow during heating (at $10^{\circ} \mathrm{C} / \mathrm{min}$ ) against temperature in hydrated HA hydrogels at different water fractions $h_{w}$ indicated on the plot.

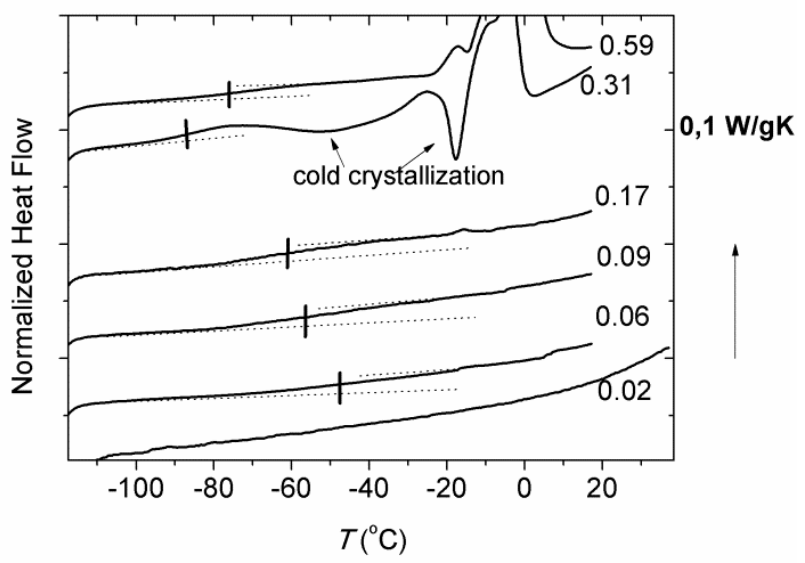

Fig.4 Normalized heat flow during heating (at $10^{\circ} \mathrm{C} / \mathrm{min}$ ) against temperature in hydrated HA hydrogels at selected water fractions $h_{w}$ indicated on the plot. The plot shows a magnification in the region of the glass transition for the selected samples. The glass transition temperature $T_{\mathrm{g}}$, is highlighted by vertical solid lines. 


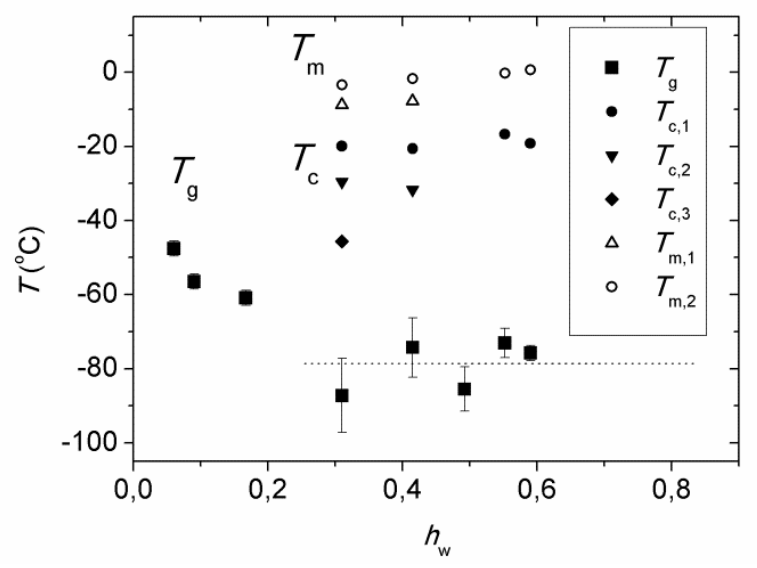

Fig.5 Characteristic temperatures, glass transition Temperature, $T_{\mathrm{g}}(\mathbf{\square})$, crystallization temperature, $T_{\mathrm{c}}(\bullet, \nabla, \diamond)$, melting temperature, $T_{\mathrm{m}}(\mathrm{O}, \triangle)$, against water fraction $h_{\mathrm{w}}$

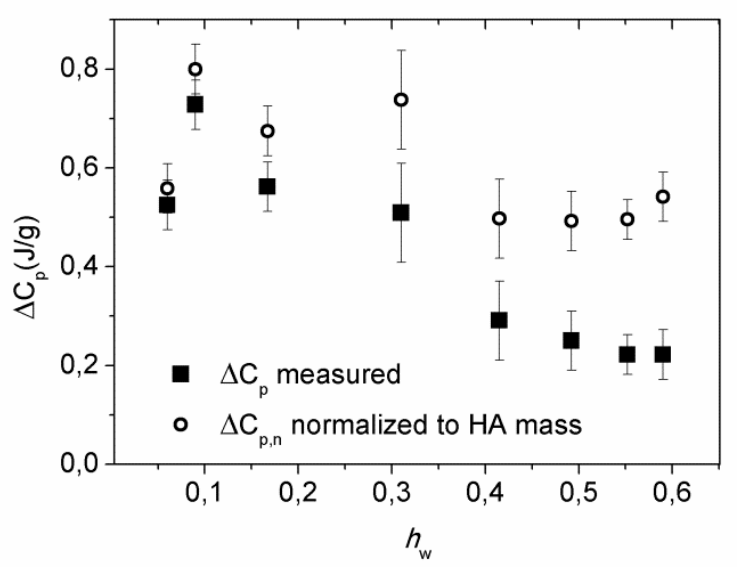

Fig.6 Heat capacity step $\Delta C_{\mathrm{p}}$ of the glass transition of the hydrated hydrogel ( $\square$ ) against water fraction $h_{\mathrm{w}}$. Normalized values to the mass of HA $(\mathrm{O}), \Delta C_{\mathrm{p}, \mathrm{n}}$, are also added to the plot. 


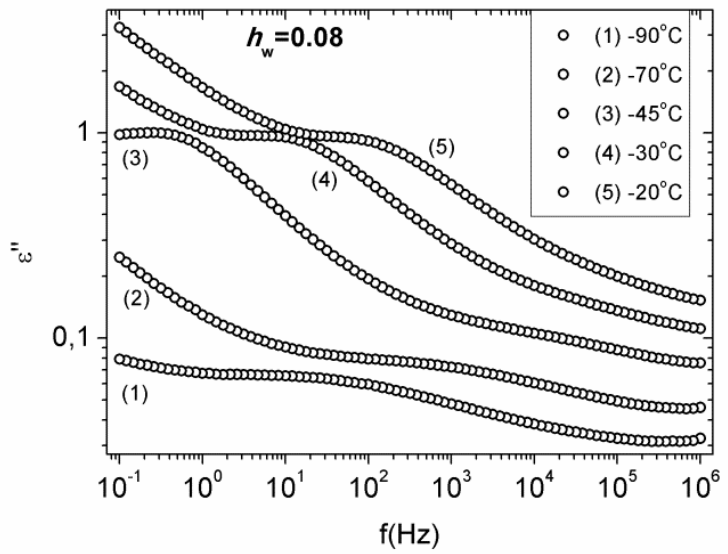

Fig.7 Imaginary part of the dielectric function, $\varepsilon^{\prime \prime}$ (dielectric loss), against frequency, at selected temperatures indicated on the plot, for a hydrated HA hydrogel of water fraction $h_{\mathrm{w}}=0.08$

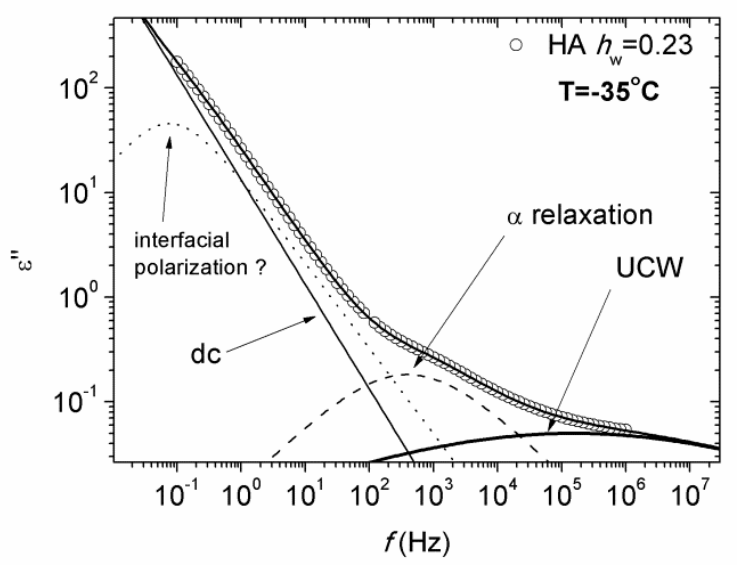

Fig.8 Dielectric loss against frequency $f(O)$ for a hydrated HA hydrogel of $h_{\mathrm{w}}=0.23$, at temperature $T=-35^{\circ} \mathrm{C}$. Solid, dotted and dashed lines correspond to contributions to dielectric loss calculated by fitting of the data by a sum of Cole-Cole functions and a conductivity term. All of the contributions are highlighted in the plot and labeled by arrows. The solid line through the experimental data corresponds to the sum of the contributions. 


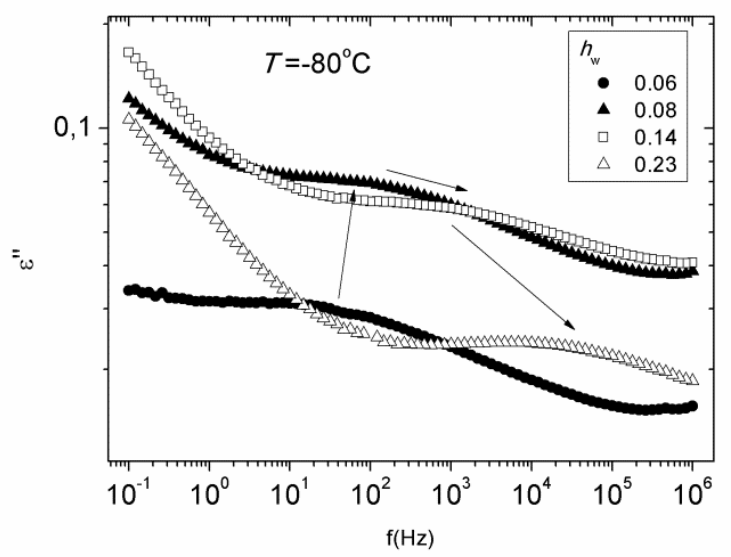

Fig.9 Dielectric loss, $\varepsilon^{\prime \prime}$, against frequency, at temperature $T=-80^{\circ} \mathrm{C}$, for hydrated HA hydrogels of water fraction values $h_{\mathrm{w}}=0.06(\bullet), 0.08(\mathbf{\Delta}), 0.14(\square)$ and $0.23(\triangle)$. The arrows follow the movement of the peak maximum in terms of increasing hydration level.

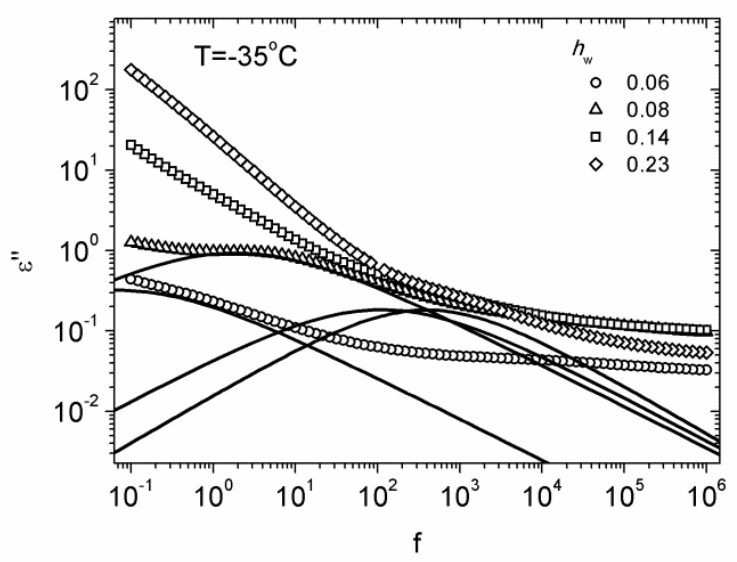

Fig.10 Dielectric loss, $\varepsilon^{\prime \prime}$, against frequency, at temperature $T=-35^{\circ} \mathrm{C}$, for hydrated HA hydrogels of water fraction values $h_{\mathrm{w}}=0.06(\bigcirc), 0.08(\triangle), 0.14(\square)$ and $0.23(\diamond)$. The solid lines are contributions of the $\alpha$ relaxation to dielectric loss, as calculated by fitting of the experimental data to a sum of Cole-Cole function and a conductivity term. 


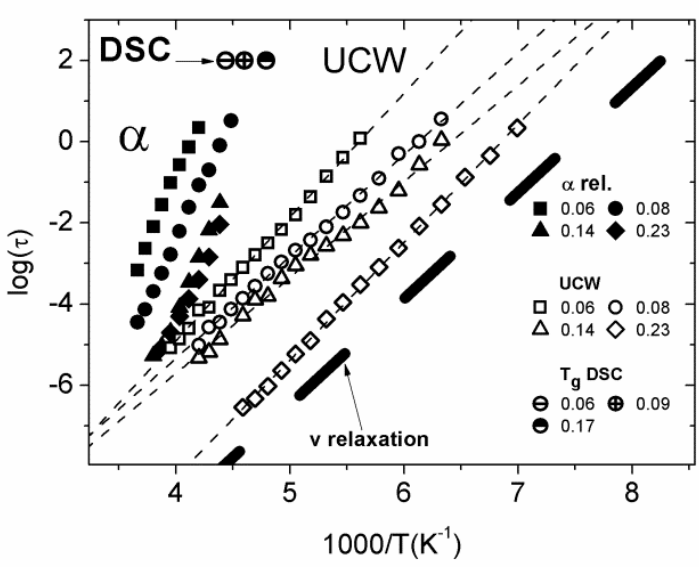

Fig.11 Temperature dependence of the relaxation times for the $\alpha$ relaxation of the hydrated HA hydrogels of water fraction $h_{\mathrm{w}}=0.06(\boldsymbol{\nabla}), 0.08(\bullet), 0.14(\boldsymbol{\Delta})$ and 0.23 $(\bullet)$ and the secondary relaxation of uncrystallized water (UCW) for $h_{\mathrm{w}}=0.06(\square)$, $0.08(\bigcirc), 0.14(\triangle)$ and $0.23(\diamond)$. The dashed lines through experimental data are fits to the Arrhenius equation. The thick dashed line corresponds to the trace of the $v$ relaxation of water [23].

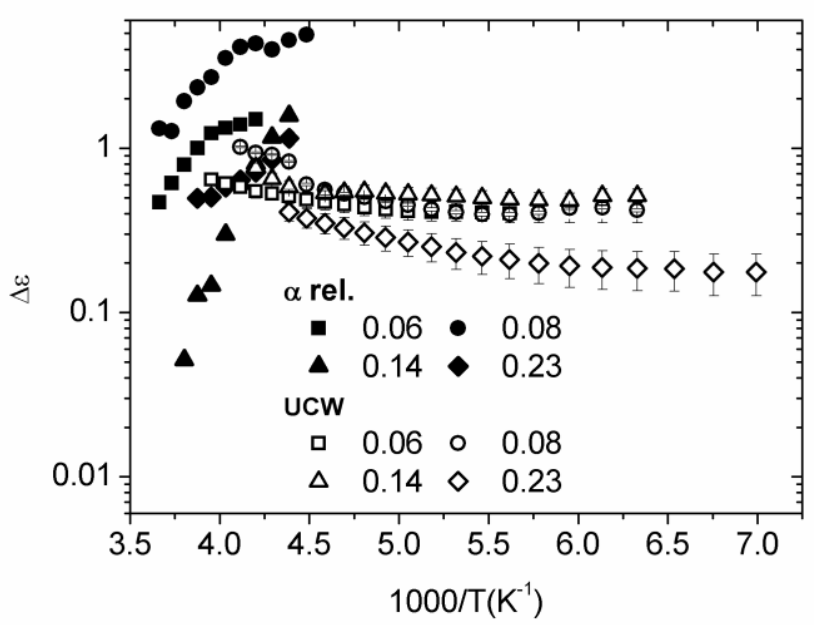

Fig.12 Relaxation strength $\Delta \varepsilon$ for the $\alpha$ relaxation of the hydrated HA hydrogels of water fraction values $h_{\mathrm{w}}=0.06(\boldsymbol{\bullet}), 0.08(\bullet), 0.14(\boldsymbol{\Delta})$ and $0.23(\bullet)$ and the secondary relaxation of uncrystallized water $(\mathrm{UCW})$ for $h_{\mathrm{w}}=0.06(\square), 0.08(O), 0.14(\triangle)$ and $0.23(\diamond)$ 


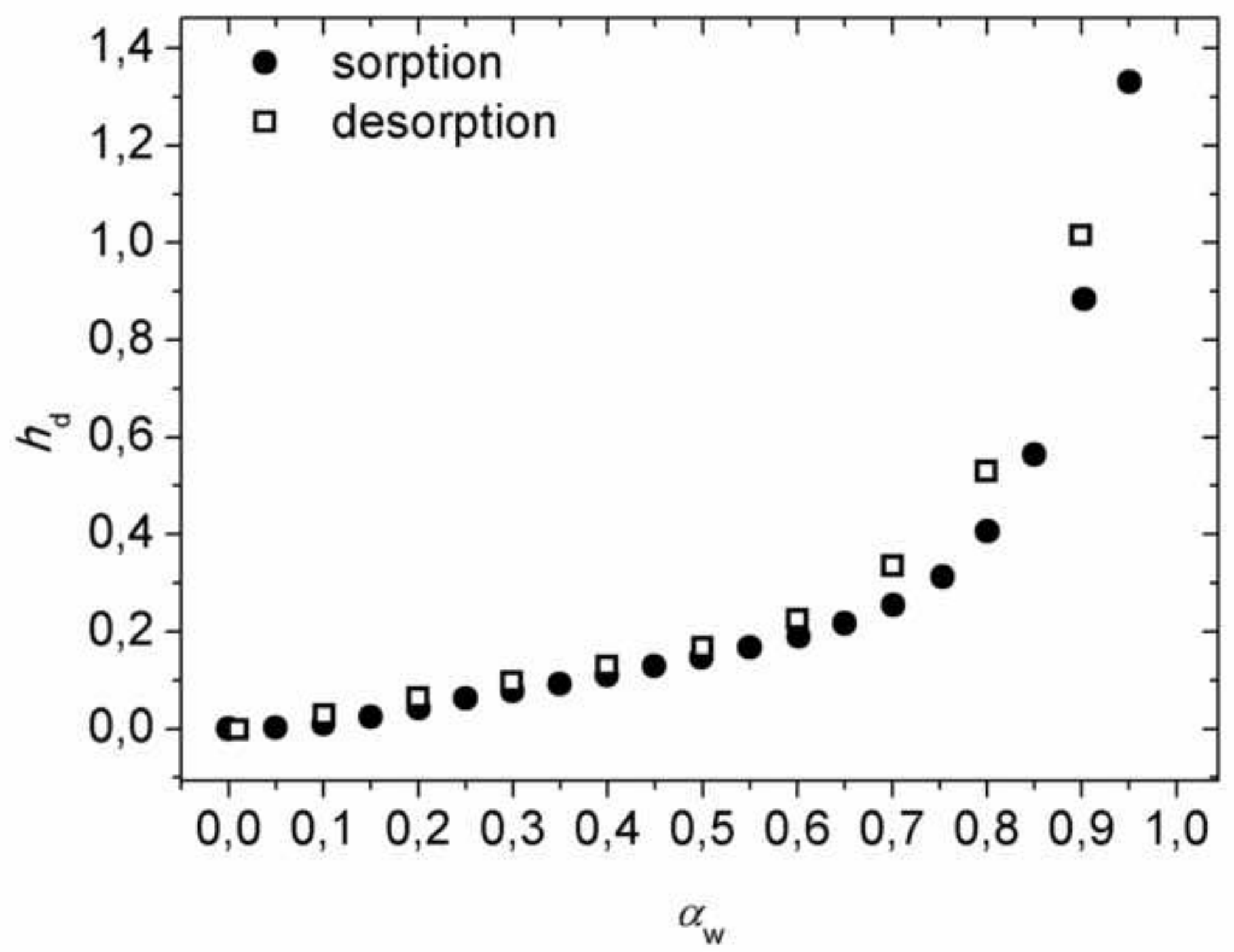




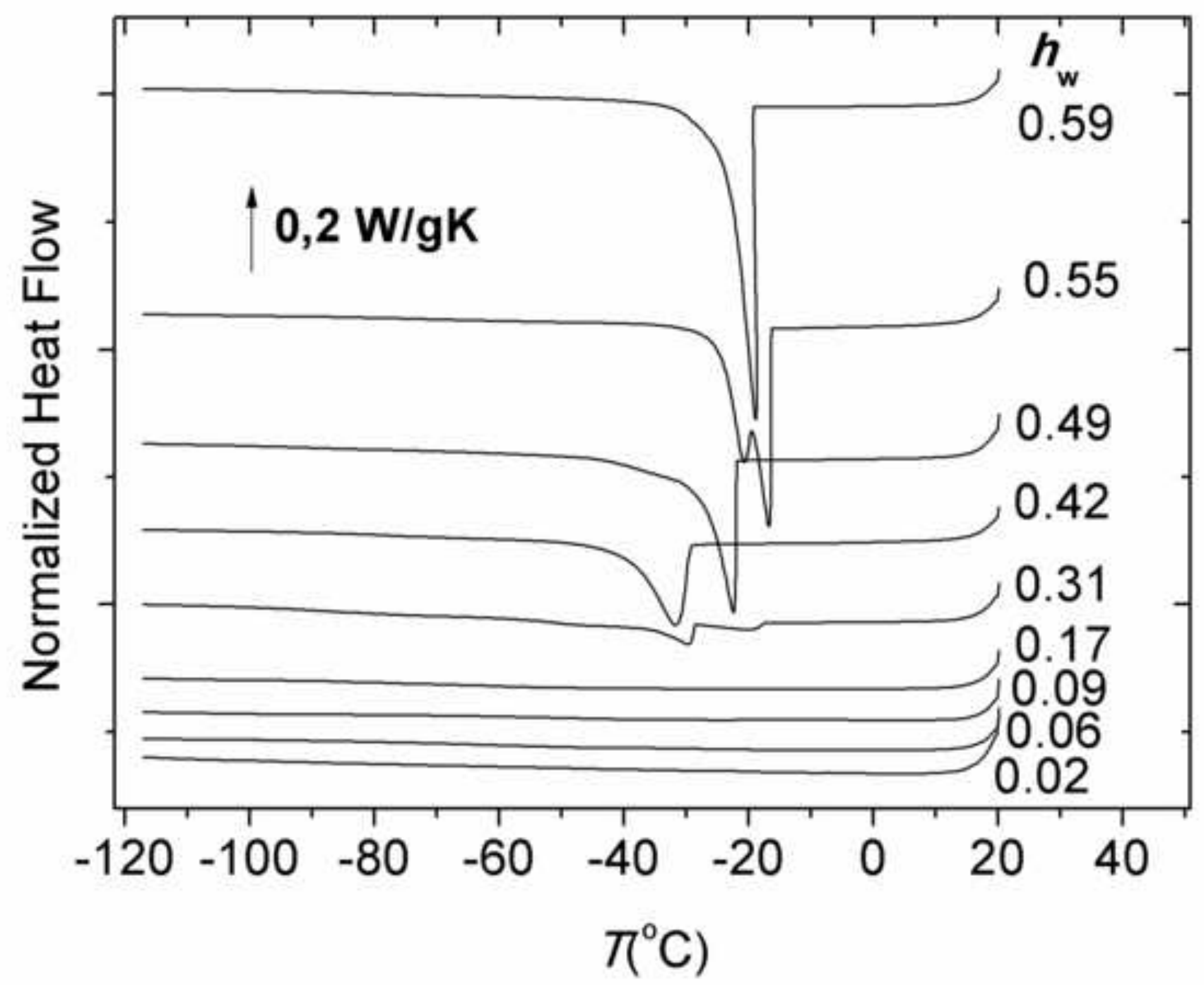




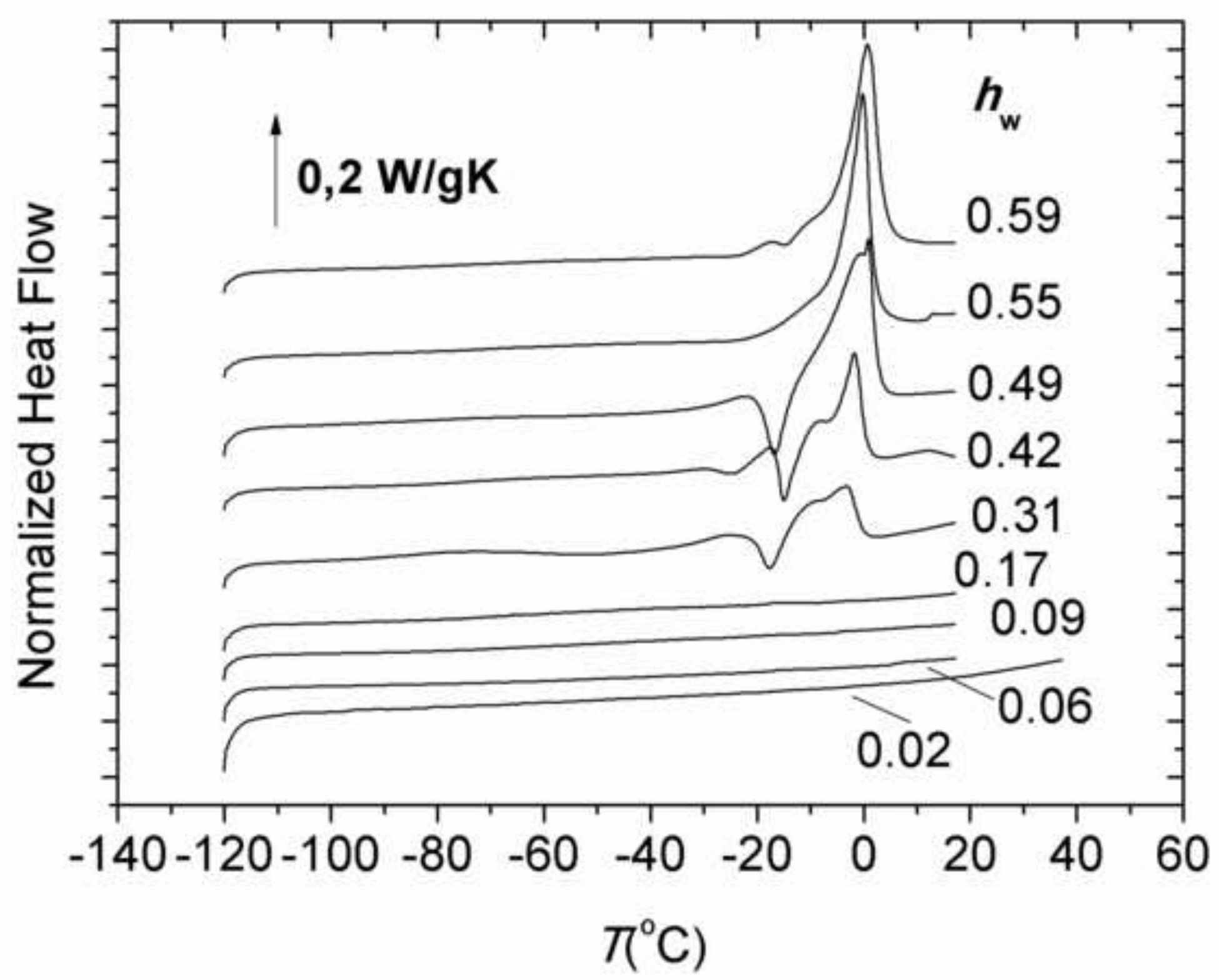




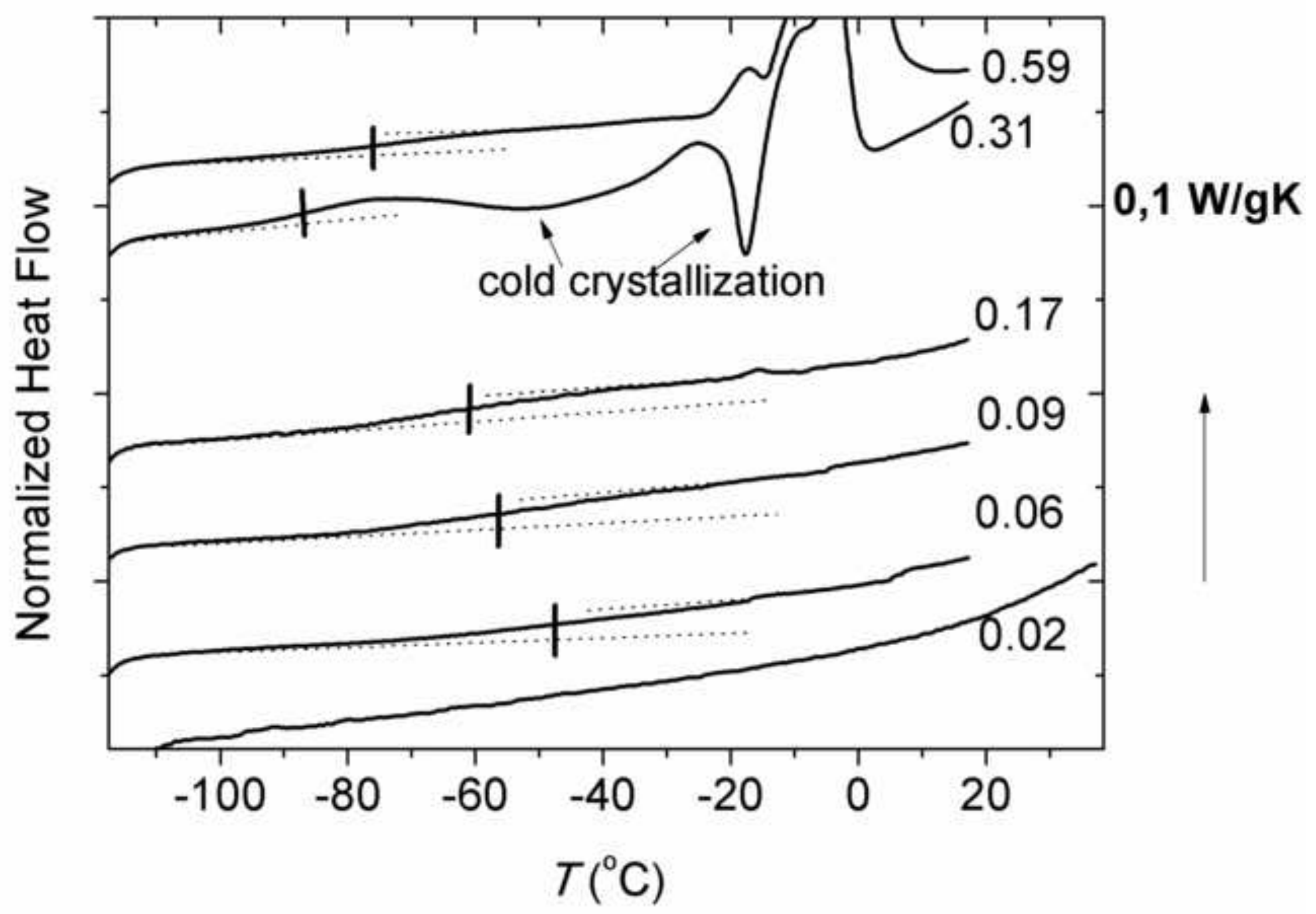




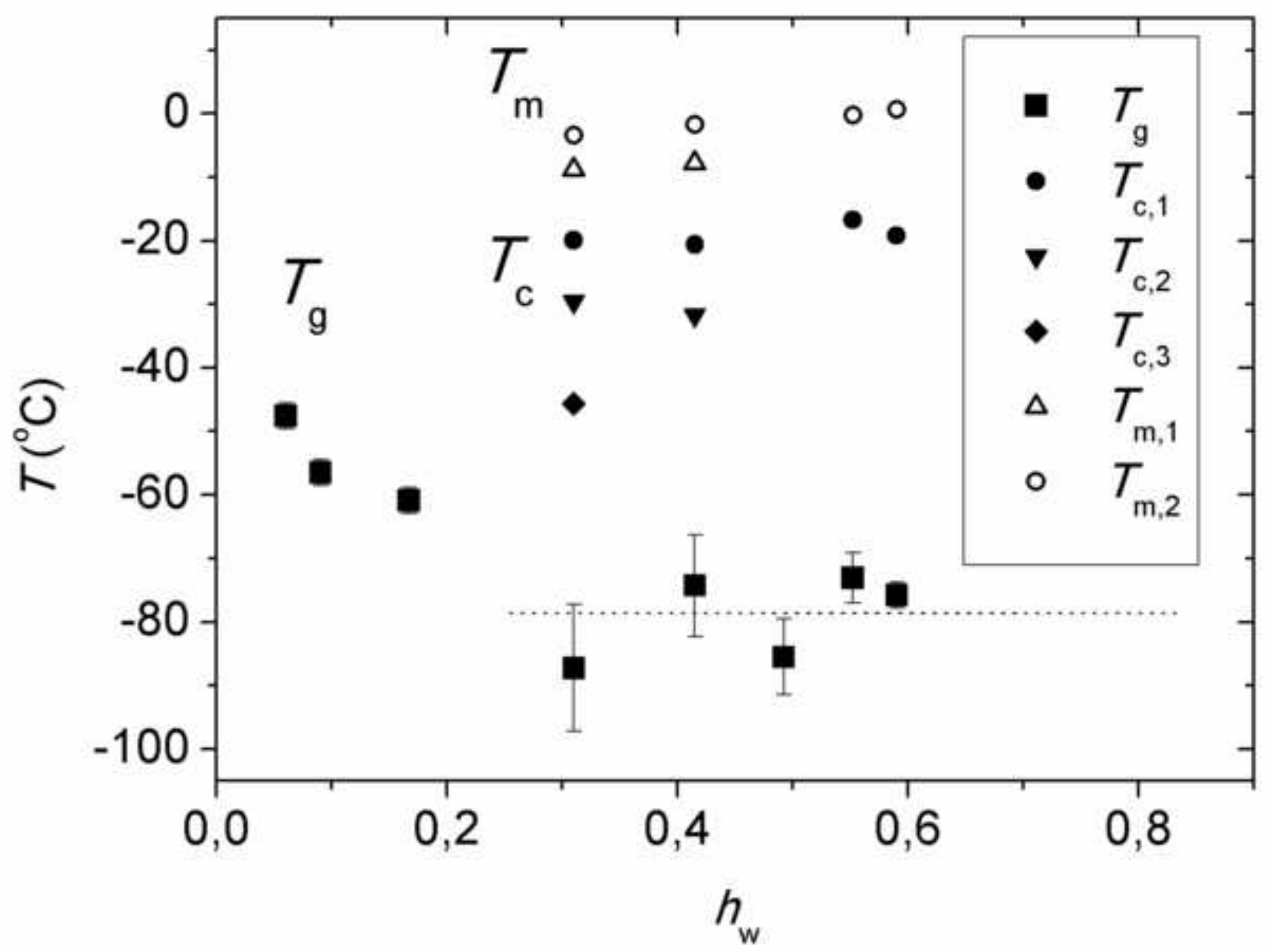




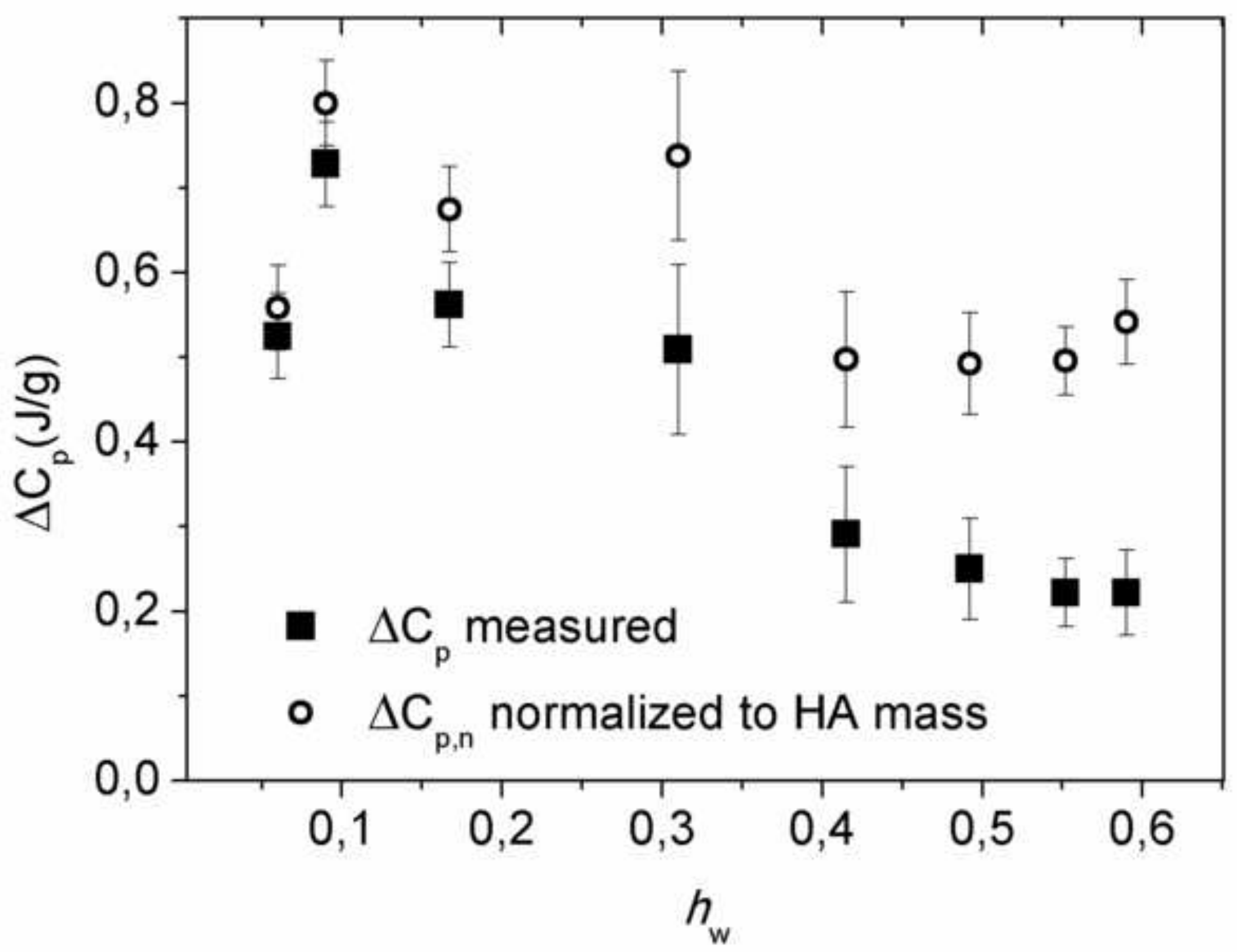




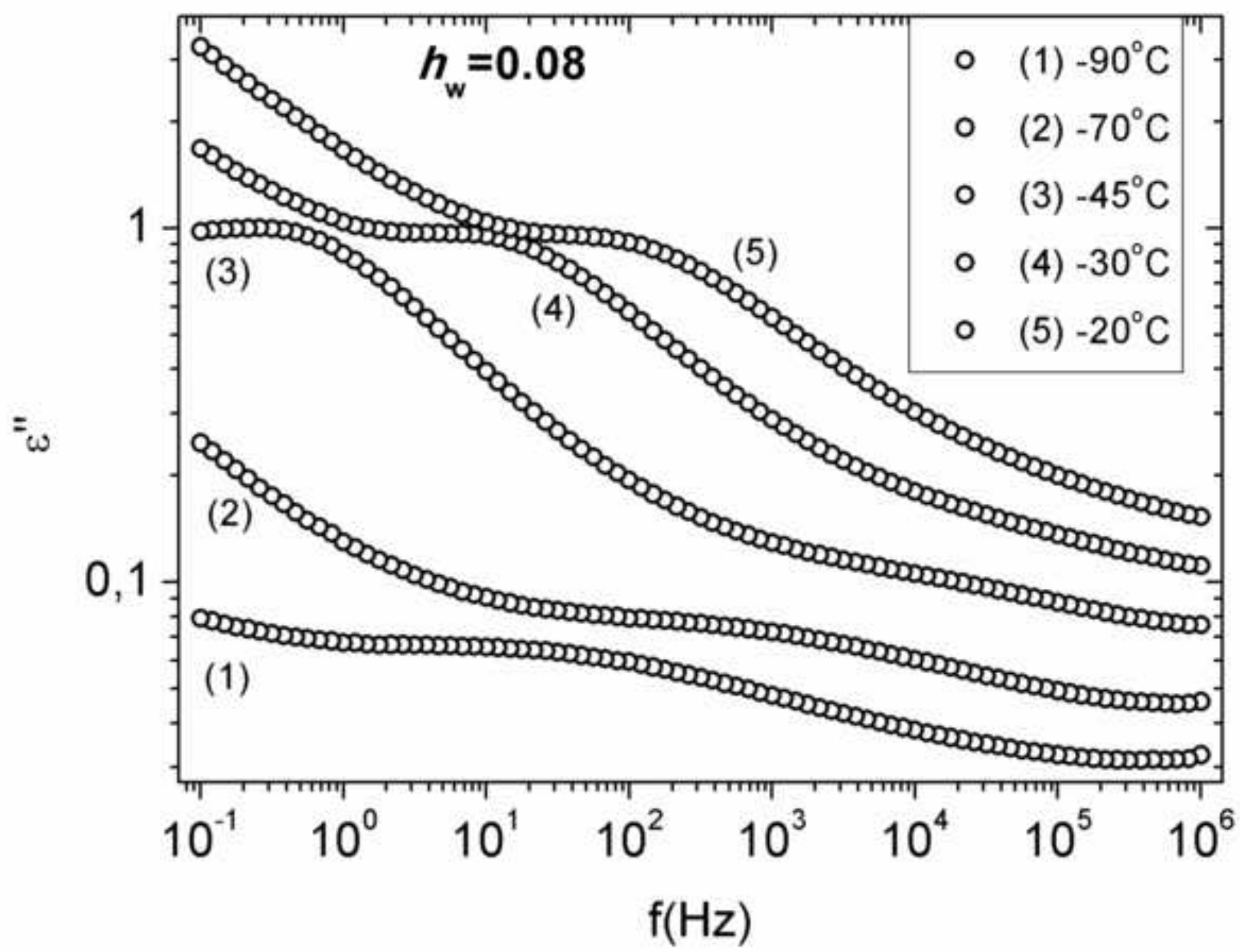




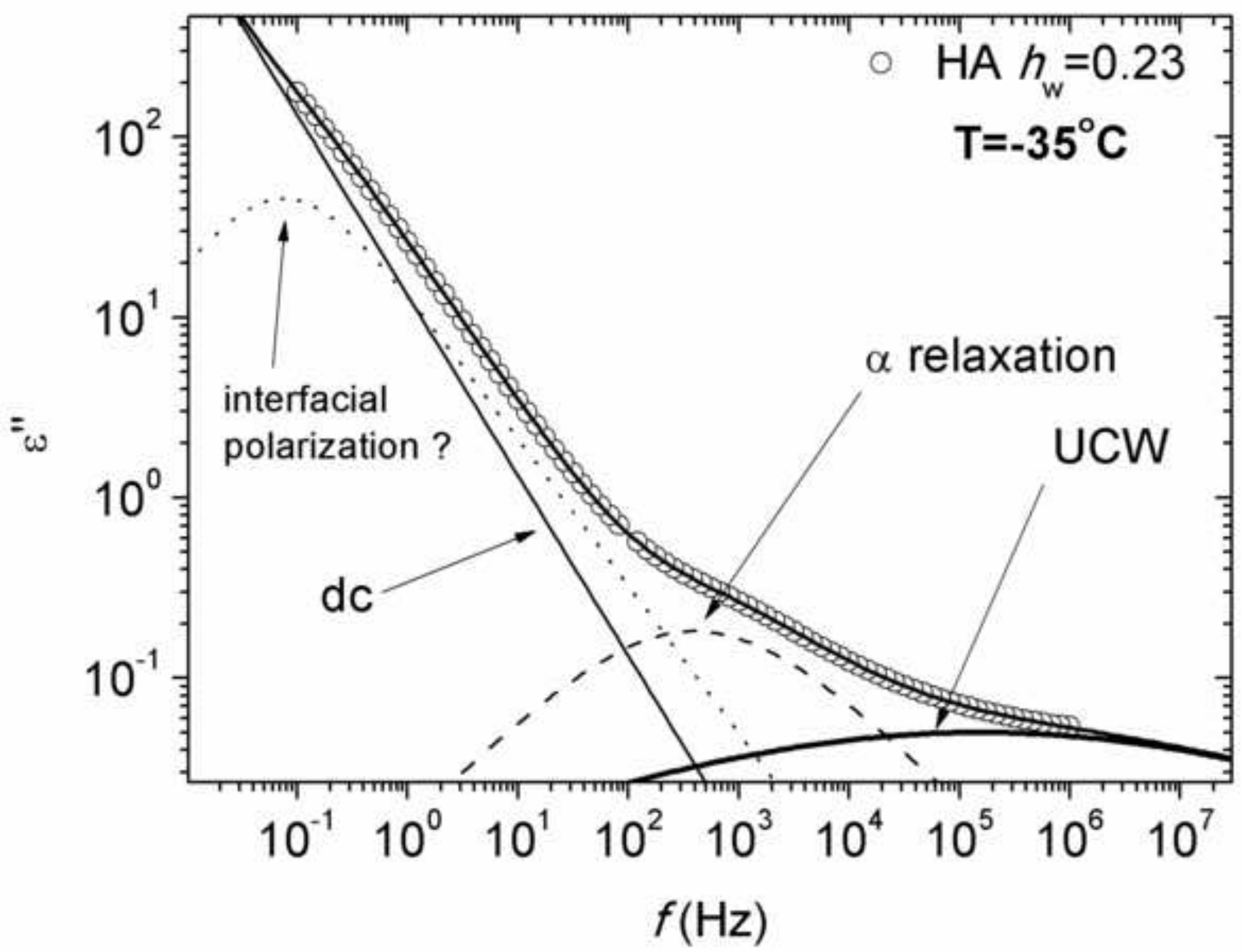




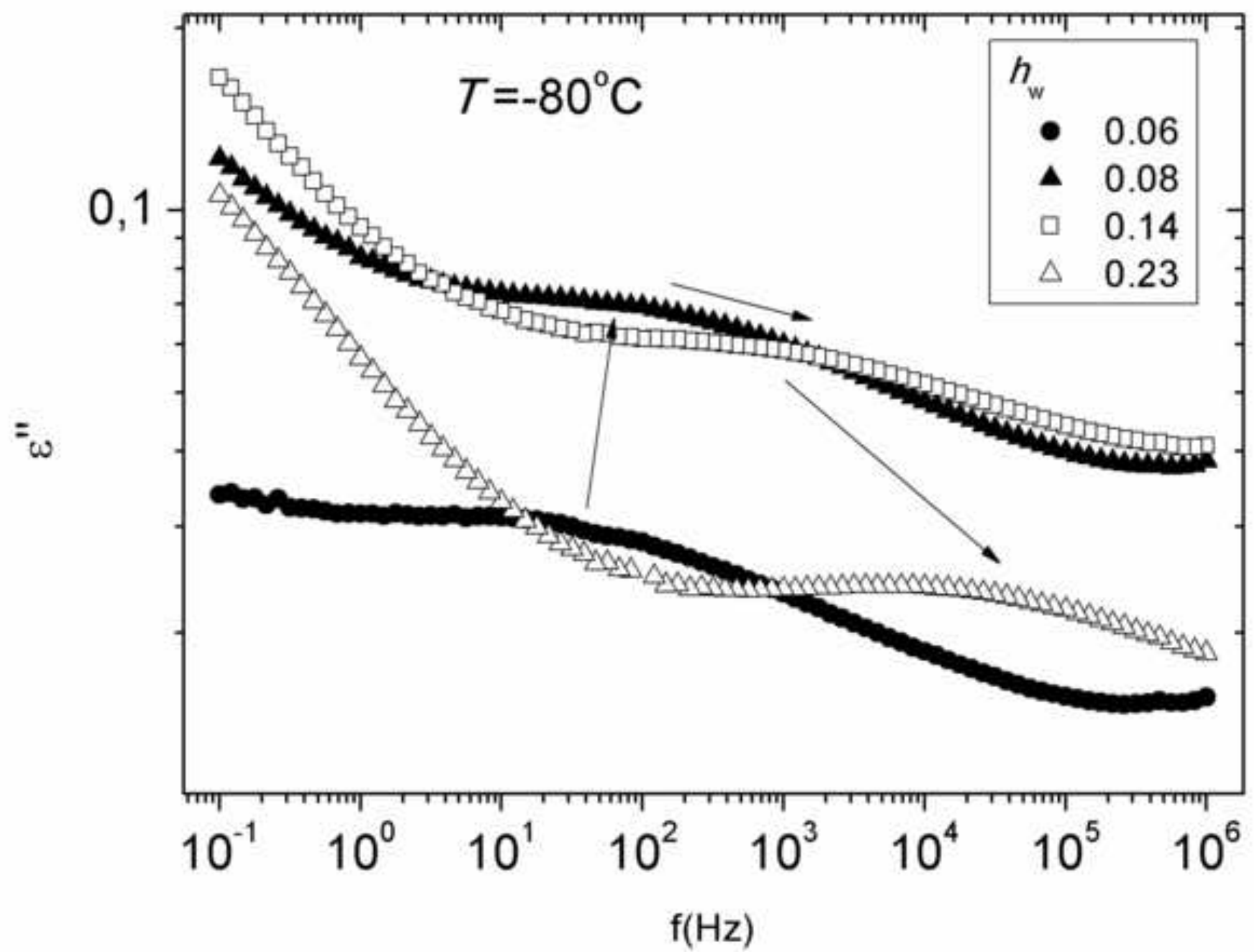




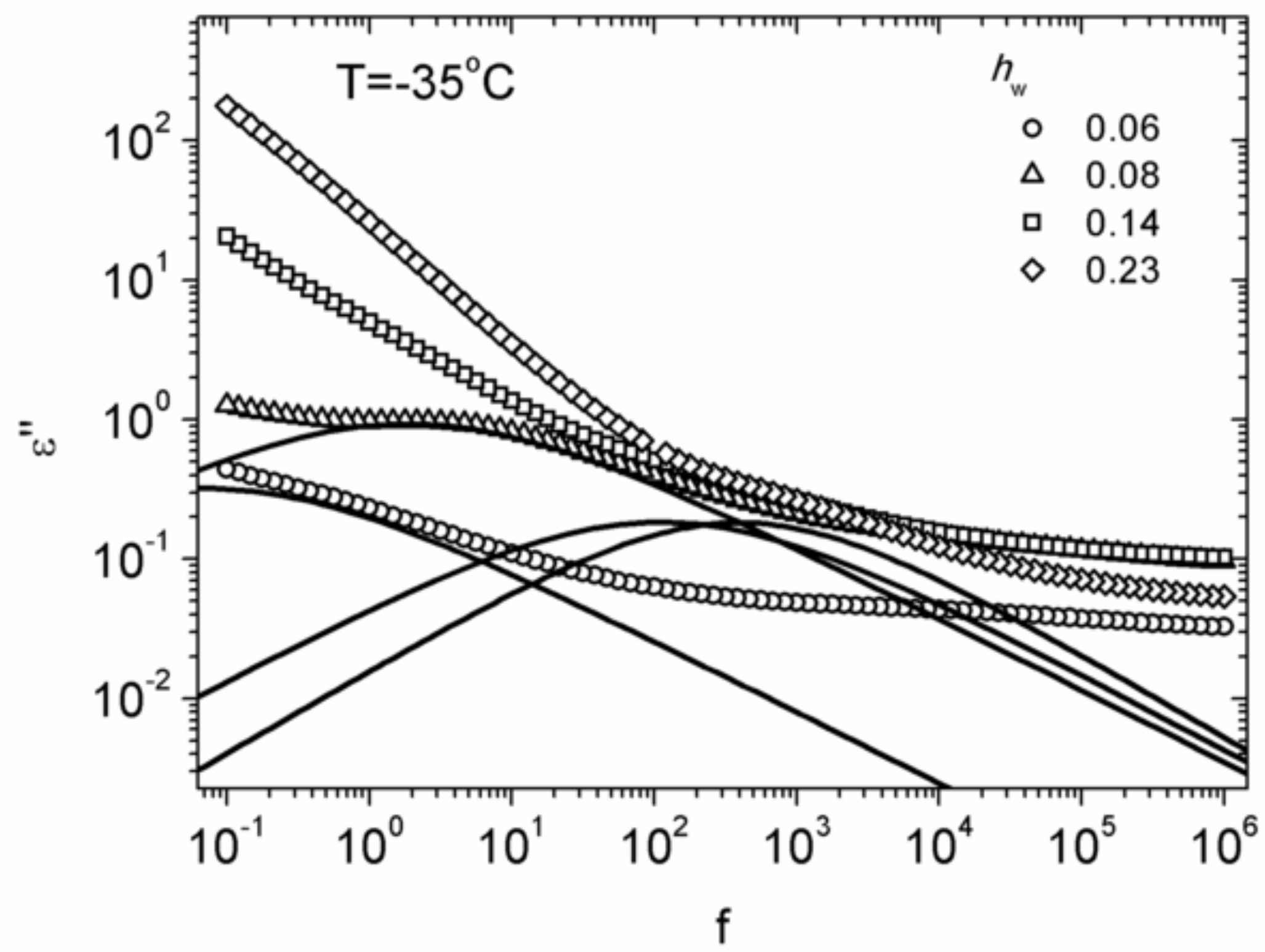




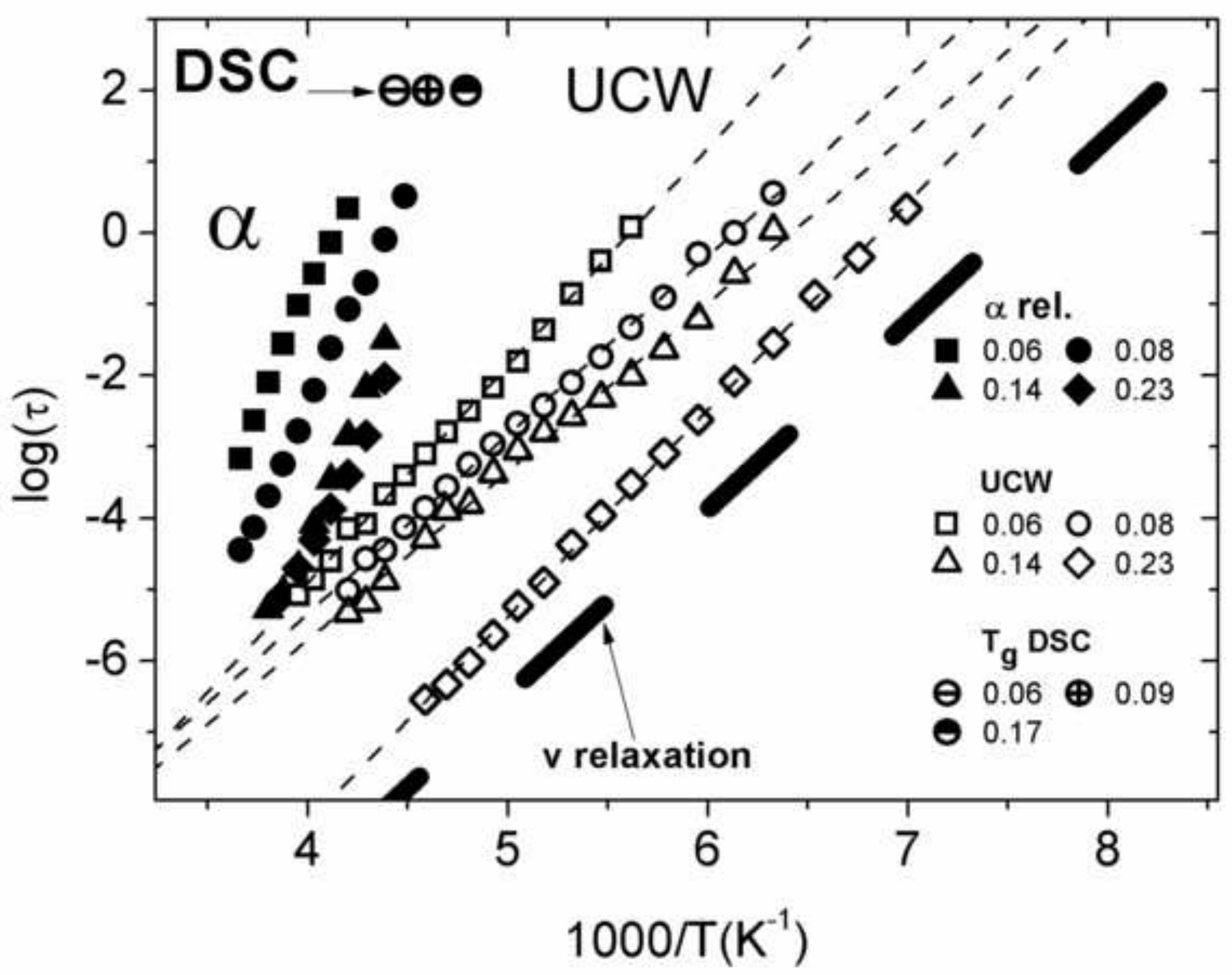




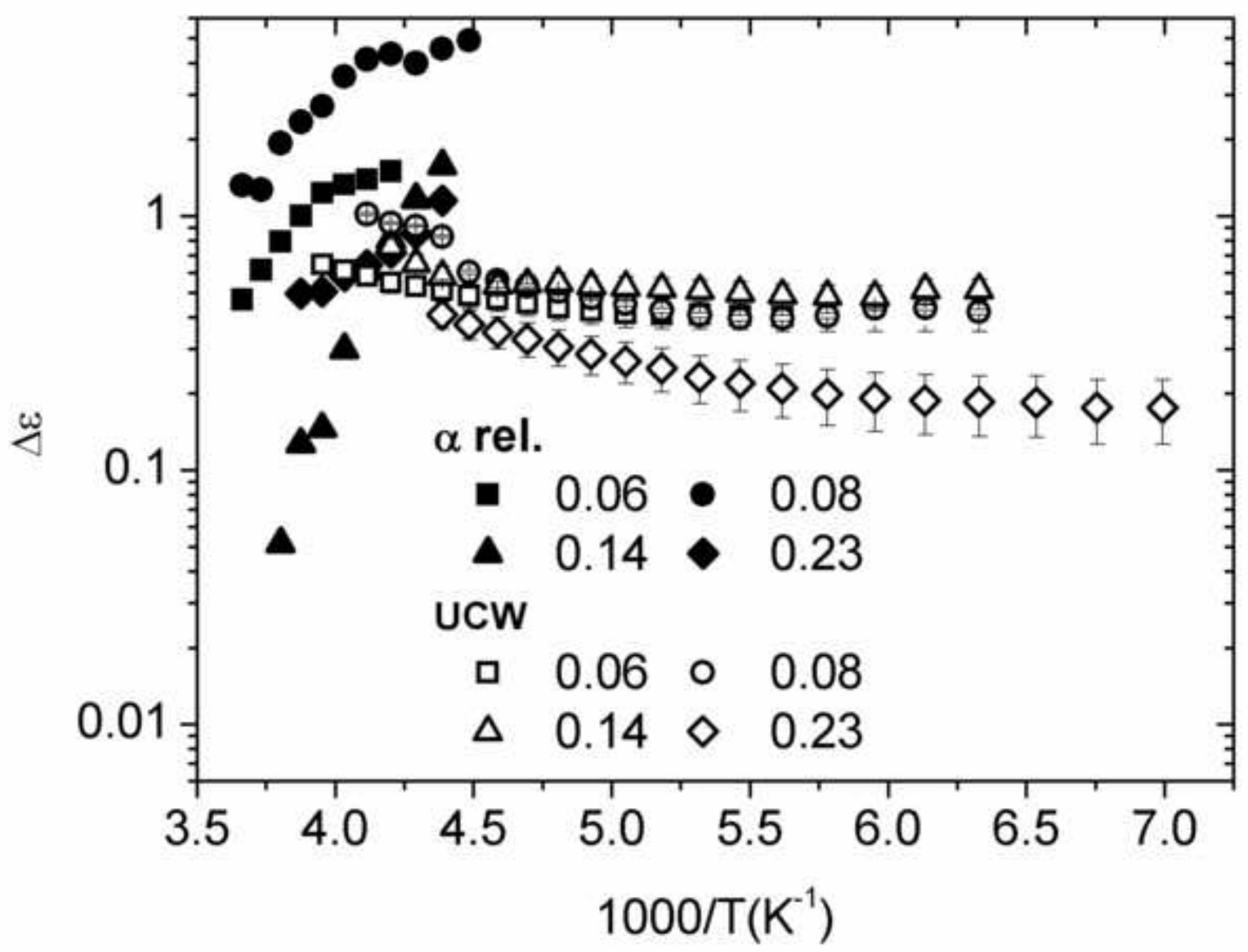

